\title{
Intensely Luminescent Alkynyl-Phosphine Gold(I)-Copper(l) Complexes: Synthesis, Characterization, Photophysical, and Computational Studies
}

\author{
Igor O. Koshevoy, ${ }^{,, \dagger}$ Yi-Chih Lin, ${ }^{\ddagger}$ Antti J. Karttunen, ${ }^{\dagger}$ Pi-Tai Chou, ${ }^{, \neq}{ }^{, \neq}$Pirjo Vainiotalo, ${ }^{\dagger}$ \\ Sergey P. Tunik, ${ }^{,}$Matti Haukka, ${ }^{\dagger}$ and Tapani A. Pakkanen ${ }^{*, \dagger}$ \\ Department of Chemistry, University of Joensuu, FI-80101, Joensuu, Finland, Department of \\ Chemistry, National Taiwan University, Taipei 106, Taiwan, and Department of Chemistry, \\ St.-Petersburg State University, Universitetskii pr. 26, 198504, St.-Petersburg, Russia
}

Received October 17, 2008

\begin{abstract}
The reactions between the diphosphino-alkynyl gold complexes $\left(\mathrm{XC}_{6} \mathrm{H}_{4} \mathrm{C}_{2} \mathrm{Au}\right) \mathrm{PR}_{2}-\mathrm{C}_{6} \mathrm{H}_{4}-\mathrm{PR}_{2}\left(\mathrm{AuC}_{2} \mathrm{C}_{6} \mathrm{H}_{4} \mathrm{X}\right)$ with $\mathrm{Cu}^{+}$lead to the formation of a family of heterometallic clusters of the general formula $\left[\left\{\mathrm{Au}_{3} \mathrm{Cu}_{2}\left(\mathrm{C}_{2} \mathrm{C}_{6} \mathrm{H}_{4} \mathrm{X}\right)_{6}\right\}\right.$ $\left.\mathrm{Au}_{3}\left(\mathrm{PR}_{2} \mathrm{C}_{6} \mathrm{H}_{4} \mathrm{PR}_{2}\right)_{3}\right]\left[\mathrm{PF}_{6}\right]_{2}\left(\mathrm{X}=\mathrm{NO}_{2}, \mathrm{H}, \mathrm{OMe}, \mathrm{NMe}_{2} ; \mathrm{R}=\mathrm{C}_{6} \mathrm{H}_{5}, \mathrm{NC}_{4} \mathrm{H}_{4}\right)$. These complexes adopt the same structural pattern and consist of a heterometallic alkynyl cluster $\left[\mathrm{Au}_{3} \mathrm{Cu}_{2}\left(\mathrm{C}_{2} \mathrm{C}_{6} \mathrm{H}_{4} \mathrm{X}\right)_{6}\right]^{-}$"wrapped" by the cationic $\left[\mathrm{Au}_{3}\left(\mathrm{PR}_{2} \mathrm{C}_{6} \mathrm{H}_{4} \mathrm{PR}_{2}\right)_{3}\right]^{3+}$ "belt". The novel compounds were characterized by NMR spectroscopy and ESI-MS measurements. A systematic study of their luminescence properties revealed efficient room-temperature phosphorescence in solution with remarkably weak quenching by molecular oxygen. The photophysical experiments demonstrate that the increase in the electron donor ability of the alkynyl ligands and the electron-withdrawing character of the diphosphines results in the bathochromic shift of emission maxima (in the 576-686 nm range) and a decrease in the luminescence quantum yield. The electronic structure calculations showed that variations of $\mathrm{X}$ or $\mathrm{R}$ substituents have very little effect on the structural parameters but display a significant influence on the electronic properties of the clusters and characteristics of luminescence. The metal-centered triplet emission within the heterometallic alkynyl cluster is suggested to play a key role in the observed phosphorescence.
\end{abstract}

\section{Introduction}

Luminescent $\mathrm{d}^{10}$ transition metal complexes attract increasing attention because of their rich photophysical and photochemical properties and possible practical applications, for example, in OLED display technology or as dopant emitters. ${ }^{1}$ In addition, the chemistry of the gold(I) compounds is of considerable interest due to their unique feature-formation of the secondary bonds through inter- or intramolecular

* To whom correspondence should be addressed. E-mail: igor.koshevoy@ joensuu.fi (I.O.K.); tapani.pakkanen@joensuu.fi (T.A.P.); chop@ntu.edu.tw (P.-TC.)

University of Joensuu.

$\doteqdot$ National Taiwan University.

$\S$ St.-Petersburg State University.

(1) (a) Ma, Y.; Che, C.-M.; Chao, H.-Y.; Zhou, X.; Chan, W.-H.; Shen, J. Adv. Mater. 1999, 11, 852-857. (b) Zhang, Q.; Zhou, Q.; Cheng, Y.; Wang, L.; Ma, D.; Jing, X.; Wang, F. Adv. Mater. 2004, 16, 432436. (c) Fave, C.; Cho, T.-Y.; Hissler, M.; Chen, C.-W.; Luh, T.-Y.; Wu, C.-C.; Reau, R. J. Am. Chem. Soc. 2003, 125, 9254-9255. (d) Baldo, M. A.; Thompson, M. E.; Forrest, S. R. Pure Appl. Chem. 1999, 71, 2095-2106. (e) Jia, W. L.; McCormick, T.; Tao, Y.; Lu, J.-P.; Wang, S. Inorg. Chem. 2005, 44, 5706-5712. aurophilic interactions. ${ }^{2}$ The ability of gold(I) ions to form heterometallophilic $\mathrm{Au} \cdots \mathrm{M}$ bonding is of particular importance because of the influence this interaction exerts onto the molecular structure and physical properties (e.g., onto luminescence) of the heterometallic aggregates. ${ }^{3}$

Among the $\mathrm{Au}(\mathrm{I})$ complexes, phosphino-alkynyls represent a specific class of organogold compounds, which are able to build up supramolecular architectures based on the

(2) (a) Forward, J. M.; Fackler, J. P. J.; Assefa, Z. In Optoelectronic Properties of Inorganic Compounds; Roundhill, D. M., Fackler, J. P. J., Eds.; Plenum: New York, 1999; pp 195-231. (b) Laguna, A. In Gold: Chemistry, Biochemistry and Technology; Schmidbauer, H.; Eds.; Wiley: Chichester, U.K., 1999; pp 349-429. (c) Dyson, P. J.; Mingos, D. M. P. In Gold: Chemistry, Biochemistry and Technology; Schmidbauer, H., Eds.; Wiley: Chichester, U. K. 1999; pp 511-557. (d) Gimeno, M. C.; Laguna, A. In Comprehensive Coordination Chemistry II; McCleverty, J. A., Meyer, T. J., Eds.; Elsevier: New York, 2003; Vol. 6, pp 911-1145. (e) Schmidbaur, H.; Schier, A. Chem. Soc. Rev. 2008, 37, 1931-1951. (f) Yam, V. W.-W.; Cheng, E. C.-C. Chem. Soc. Rev. 2008, 37, 1806-1813.

(3) Fernandez, E. J.; Laguna, A.; Lopez-de-Luzuriaga, J. M. Dalton Trans. 2007, 1969-1981. 
metallophilic interactions and the ability of the alkynyl units to function as linear rigid-rod bridging building blocks. The supramolecular aggregates are stabilized by ancillary phosphine ligands that allow for the preparation of a number of the luminescent heterometallic polynuclear complexes. ${ }^{4-6}$

In our recent studies, ${ }^{5,6}$ we reported an investigation of the $\mathrm{Cu}(\mathrm{I})$-promoted assembly of the $\mathrm{Au}-\mathrm{Cu}$ alkynyl clusters supported by the $\left[\mathrm{Au}_{3}(\text { diphospine })_{3}\right]^{3+}$ triangular "belts" (diphosphine $\left.=\mathrm{PPh}_{2}\left(\mathrm{C}_{6} \mathrm{H}_{4}\right)_{n} \mathrm{PPh}_{2} ; n=1,2,3\right)$. Variations in the length of the diphosphine ligand spacers resulted in the successful synthesis of a series of heterometallic complexes of the general formula $\left[\left\{\mathrm{Au}_{x} \mathrm{Cu}_{y}\left(\mathrm{C}_{2} \mathrm{Ph}\right)_{2 x}\right\}\right.$ $\left.\mathrm{Au}_{3}\left\{\mathrm{PPh}_{2}-\left(\mathrm{C}_{6} \mathrm{H}_{4}\right)_{n}-\mathrm{PPh}_{2}\right\}_{3}\right]^{3+(y-x)}\left(n=1,2,3 ; x={ }_{n}+\right.$ 1) $(n+2) / 2 ; y=n(n+1))$. These compounds display very similar structural patterns and contain the central heterometallic clusters $\left\{\mathrm{Au}_{x} \mathrm{Cu}_{y}\left(\mathrm{C}_{2} \mathrm{Ph}\right)_{2 x}\right\}$ wrapped about by the tricationic $\left[\mathrm{Au}_{3}(\mathrm{PP})_{3}\right]^{3+}$ "belts", anchored to the central parts by the $\mathrm{Au}-\mathrm{Au}$ bonds. The complexes obtained display very intense room-temperature long-wavelength luminescence both in solution and in the solid state with a maximum quantum yield of $92 \%(n=1)$. However, it was experimentally demonstrated that the increase in the size of the aggregates leads to the decrease in photostability and luminescence efficiency that was supported by the computational results, which also indicated that the phosphorescence observed originates from the metal-centered transitions within the heterometallic $\mathrm{Au}-\mathrm{Cu}$ cores. ${ }^{6}$ This prompted us to experimental and theoretical studies related to modification of the electronic structure of this type of compounds via variation of the coordination environment. The most stable and effective luminophore $\left[\left\{\mathrm{Au}_{3} \mathrm{Cu}_{2}\left(\mathrm{C}_{2} \mathrm{Ph}\right)_{6}\right\} \mathrm{Au}_{3}\left\{\mathrm{PPh}_{2}-\right.\right.$ $\left.\left.\mathrm{C}_{6} \mathrm{H}_{4}-\mathrm{PPh}_{2}\right\}_{3}\right]^{2+}$ was chosen as a parent compound to prepare its analogues containing substituted phenylalkynes and diphosphines with different electron-donor properties and to study the effect of these variations on the luminescence properties of these supramolecular aggregates.

\section{Experimental Section}

General Comments. 1,4-Bis(diphenylphosphino)benzene ${ }^{7}$ and 1,4-bis(dichlorophosphino)benzene ${ }^{8}$ were synthesized according to published procedures. Complexes $\left\{\mathrm{Au}\left(1-\mathrm{C}_{2}-4-\mathrm{X}_{-} \mathrm{C}_{6} \mathrm{H}_{4}\right)\right\}_{n}(\mathrm{X}=$ $\left.\mathrm{NO}_{2}, \mathrm{OMe}, \mathrm{NMe}_{2}\right)$ were prepared analogously to $\left(\mathrm{AuC}_{2} \mathrm{Ph}\right)_{n}{ }^{9}$ using commercially available alkynes $1-\mathrm{HC}_{2}-4-\mathrm{X}-\mathrm{C}_{6} \mathrm{H}_{4}$. Tetrahydrofuran was distilled over Na-benzophenoneketyl under a nitrogen atmosphere prior to use. Other reagents and solvents were used as

(4) (a) Wei, Q.-H.; Yin, G.-Q.; Zhang, L.-Y.; Shi, L.-X.; Mao, Z.-W.; Chen, Z.-N. Inorg. Chem. 2004, 43, 3484-3491. (b) Wei, Q.-H.; Zhang, L.-Y.; Yin, G.-Q.; Shi, L.-X.; Chen, Z.-N. J. Am. Chem. Soc. 2004, 126, 9940-9941. (c) Yin, G.-Q.; Wei, Q.-H.; Zhang, L.-Y.; Chen, Z.N. Organometallics 2006, 25, 580-587. (d) de la Riva, H.; Nieuwhuyzen, M.; Fierro, C. M.; Raithby, P. R.; Male, L.; Lagunas, M. C. Inorg. Chem. 2006, 45, 1418-1420.

(5) Koshevoy, I. O.; Koskinen, L.; Haukka, M.; Tunik, S. P.; Serdobintsev, P. Y.; Melnikov, A. S.; Pakkanen, T. A. Angew. Chem., Int. Ed. 2008, 47, 3942-3945.

(6) Koshevoy, I. O.; Karttunen, A. J.; Tunik, S. P.; Haukka, M.; Selivanov, S. I.; Melnikov, A. S.; Serdobintsev, P. Y.; Khodorkovskiy, M. A.; Pakkanen, T. A. Inorg. Chem. 2008, 47, 9478-9488.

(7) Baldwin, R. A.; Cheng, M. T. J. Org. Chem. 1967, 32, 1572-1577.

(8) Evleth, E. M. J.; Freeman, L. D.; Wagner, R. I. J. Org. Chem. 1962, 27, 2192-2197.

(9) Coates, G. E.; Parkin, C. J. Chem. Soc. 1962, 3220-3226. received. The solution ${ }^{1} \mathrm{H},{ }^{13} \mathrm{C}$, and ${ }^{31} \mathrm{P}$ NMR spectra were recorded on Bruker Avance 400 and Bruker DPX 300 spectrometers. Mass spectra were measured on a Bruker APEX-Qe ESI FT-ICR instrument, in the $\mathrm{ESI}^{+}$mode. Microanalyses were carried out in the analytical laboratories of St.-Petersburg State University and the University of Joensuu.

$\mathbf{P}\left(\mathrm{NC}_{4} \mathbf{H}_{4}\right)_{2} \mathbf{C}_{6} \mathbf{H}_{4} \mathbf{P}\left(\mathrm{NC}_{4} \mathrm{H}_{4}\right)_{2}$ (1). Synthesis was carried under a nitrogen atmosphere. Pyrrole (1.6 g, $23.9 \mathrm{mmol})$ was added dropwise to a cooled $\left(-40{ }^{\circ} \mathrm{C}\right)$ solution of $n$-BuLi in hexanes $(16$ $\mathrm{cm}^{3}, 1.6 \mathrm{M}$ ). A pale suspension was stirred at $-40{ }^{\circ} \mathrm{C}$ for $30 \mathrm{~min}$, warmed to room temperature, and stirred for an additional $30 \mathrm{~min}$. Then, the reaction mixture was cooled to $-78{ }^{\circ} \mathrm{C}$ and treated dropwise with a solution of 1,4-bis(dichlorophosphino)benzene $(1.64 \mathrm{~g}, 5.85 \mathrm{mmol})$ in THF $\left(25 \mathrm{~cm}^{3}\right)$. When the addition was completed, the reaction mixture was warmed to room temperature and stirred for $1 \mathrm{~h}$. The resulting yellow-brownish solution was filtered to remove a small amount of white precipitate. The solvents were evaporated. An oily residue was extracted with $\mathrm{CH}_{2} \mathrm{Cl}_{2}(3 \times$ $\left.4 \mathrm{~cm}^{3}\right)$, and extracts were diluted with hexanes $\left(10 \mathrm{~cm}^{3}\right)$ and passed through a layer of silica $(2 \times 5 \mathrm{~cm})$. The solvents again were removed; the resulting yellow solid was washed with methanol (3 $\times 5 \mathrm{~cm}^{3}$ ) to give white material of sufficient (ca. 97\%) purity $(1.05$ $\mathrm{g}, 45 \%$ ). An analytically pure sample was obtained by additional chromatographic purification on silica (2:3 eluent $\mathrm{CH}_{2} \mathrm{Cl}_{2} /$ hexane v/v). ${ }^{31} \mathrm{P}\left\{{ }^{1} \mathrm{H}\right\}$ NMR $\left(\mathrm{CDCl}_{3} ; \delta\right): 63.2(\mathrm{~s}) .{ }^{1} \mathrm{H} \mathrm{NMR}\left(\mathrm{CDCl}_{3} ; \delta\right)$ : 7.08 ( $\left.\mathrm{m}, \mathrm{C}_{6} \mathrm{H}_{4}, 4 \mathrm{H}\right), 6.95$ (m, Pyr, 8H), 6.35 (m, Pyr, 8H). Anal. calcd for $\mathrm{C}_{22} \mathrm{H}_{20} \mathrm{~N}_{4} \mathrm{P}_{2}$ : C, 65.67; H, 5.01; N, 13.92. Found: C, 65.54; $\mathrm{H}, 5.11 ; \mathrm{N}, 13.77$.

$\left(\mathbf{A u C}_{\mathbf{2}} \mathbf{C}_{\mathbf{6}} \mathbf{H}_{\mathbf{4}} \mathbf{N O}_{2}\right)_{2} \mathbf{P P h} \mathbf{C}_{\mathbf{6}} \mathbf{H}_{\mathbf{4}} \mathbf{P P h}_{\mathbf{2}}$ (2). $\left(\mathrm{AuC}_{2} \mathrm{C}_{6} \mathrm{H}_{4} \mathrm{NO}_{2}\right)_{n}(150 \mathrm{mg}$, $0.437 \mathrm{mmol})$ was suspended in $\mathrm{CH}_{2} \mathrm{Cl}_{2}\left(10 \mathrm{~cm}^{3}\right)$, and a slight excess of 1,4-bis(diphenylphosphino)benzene (102 $\mathrm{mg}, 0.229 \mathrm{mmol}$ ) was added. The reaction mixture was stirred for $30 \mathrm{~min}$. The suspension obtained was evaporated, dissolved in $\mathrm{CHCl}_{3}$ (ca. $20 \mathrm{~cm}^{3}$ ), diluted with toluene $\left(5 \mathrm{~cm}^{3}\right)$, and passed through a layer of neutral $\mathrm{Al}_{2} \mathrm{O}_{3}$ $(1 \times 4 \mathrm{~cm})$. The solution was concentrated to a volume of ca. 7 $\mathrm{cm}^{3}$. A pale solid was separated by centrifugation, suspended in $\mathrm{CH}_{2} \mathrm{Cl}_{2}\left(2 \mathrm{~cm}^{3}\right)$, diluted with toluene $\left(4 \mathrm{~cm}^{3}\right)$, and centrifugated again. Subsequent washing with toluene $\left(5 \mathrm{~cm}^{3}\right)$ and diethyl ether $\left(3 \times 5 \mathrm{~cm}^{3}\right)$ and vacuum drying gave a pale-yellow powder $(235$ $\mathrm{mg}, 95 \%)$. An analytically pure sample was obtained by recrystallization from $\mathrm{CH}_{2} \mathrm{Cl}_{2}$ /diethyl ether at $+5{ }^{\circ} \mathrm{C} .{ }^{31} \mathrm{P}\left\{{ }^{1} \mathrm{H}\right\} \mathrm{NMR}$ $\left(\mathrm{CDCl}_{3} ; \delta\right): 41.4(\mathrm{~s}) .{ }^{1} \mathrm{H}$ NMR $\left(\mathrm{CDCl}_{3} ; \delta\right): 8.14\left(\mathrm{dm}, \mathrm{C}_{6} \mathrm{H}_{4}-\mathrm{NO}_{2}\right.$, $J(\mathrm{H}-\mathrm{H}) 8.9 \mathrm{~Hz}, 4 \mathrm{H}), 7.62-7.49(\mathrm{~m}, 28 \mathrm{H})$. Anal. calcd for $\mathrm{C}_{46} \mathrm{H}_{32} \mathrm{Au}_{2} \mathrm{~N}_{2} \mathrm{O}_{4} \mathrm{P}_{2}$ : C, 48.78; H, 2.85; N, 2.47. Found: C, 48.82; H, $2.80 ; \mathrm{N}, 2.35$.

$\left(\mathrm{AuC}_{2} \mathbf{C}_{\mathbf{6}} \mathbf{H}_{\mathbf{4}} \mathbf{O M e}\right)_{2} \mathbf{P P h}_{\mathbf{2}} \mathbf{C}_{\mathbf{6}} \mathbf{H}_{\mathbf{4}} \mathbf{P P h}_{\mathbf{2}}$ (3). $\left(\mathrm{AuC}_{2} \mathrm{C}_{6} \mathrm{H}_{4} \mathrm{OMe}\right)_{n}(150$ $\mathrm{mg}, 0.457 \mathrm{mmol})$ was suspended in $\mathrm{CH}_{2} \mathrm{Cl}_{2}\left(10 \mathrm{~cm}^{3}\right)$, and $1,4-$ bis(diphenylphosphino)benzene (106 mg, $0.238 \mathrm{mmol}$ ) was added. The reaction mixture was stirred for $30 \mathrm{~min}$. The colorless transparent solution was diluted with toluene $\left(5 \mathrm{~cm}^{3}\right)$ and passed through a layer of neutral $\mathrm{Al}_{2} \mathrm{O}_{3}(1 \times 4 \mathrm{~cm})$. The solution was concentrated to a volume of ca. $5 \mathrm{~cm}^{3}$. A white solid was separated by centrifugation, washed with toluene $\left(5 \mathrm{~cm}^{3}\right)$ and diethyl ether $\left(3 \times 5 \mathrm{~cm}^{3}\right)$, and vaccuum-dried $(232 \mathrm{mg}, 92 \%)$. An analytically pure sample was obtained by recrystallization from $\mathrm{CH}_{2} \mathrm{Cl}_{2} /$ diethyl ether at $+5{ }^{\circ} \mathrm{C} .{ }^{31} \mathrm{P}\left\{{ }^{1} \mathrm{H}\right\}$ NMR $\left(\mathrm{CDCl}_{3} ; \delta\right): 41.8$ (s). ${ }^{1} \mathrm{H}$ NMR $\left(\mathrm{CDCl}_{3} ; \delta\right): 7.62-7.47(\mathrm{~m}, 24 \mathrm{H}), 7.45\left(\mathrm{dm}, \mathrm{C}_{6} \mathrm{H}_{4}-\mathrm{OCH}_{3}, J(\mathrm{H}-\mathrm{H})\right.$ $8.2 \mathrm{~Hz}, 4 \mathrm{H}), 6.80\left(\mathrm{dm}, \mathrm{C}_{6} \mathrm{H}_{4}-\mathrm{OCH}_{3}, J(\mathrm{H}-\mathrm{H}) 8.2 \mathrm{~Hz}, 4 \mathrm{H}\right), 3.80$ (s, $\mathrm{OCH}_{3}, 6 \mathrm{H}$ ). Anal. calcd for $\mathrm{C}_{48} \mathrm{H}_{38} \mathrm{Au}_{2} \mathrm{O}_{2} \mathrm{P}_{2}$ : C, 52.28; H, 3.47. Found: C, 52.23; H, 3.46.

$\left(\mathbf{A u C}_{\mathbf{2}} \mathbf{C}_{\mathbf{6}} \mathbf{H}_{\mathbf{4}} \mathbf{N M e}_{2}\right)_{2} \mathbf{P} \mathbf{P h}_{\mathbf{2}} \mathbf{C}_{\mathbf{6}} \mathbf{H}_{\mathbf{4}} \mathbf{P P h}_{\mathbf{2}}$ (4). $\left(\mathrm{AuC}_{2} \mathrm{C}_{6} \mathrm{H}_{4} \mathrm{NMe}_{2}\right)_{n}(150$ $\mathrm{mg}, 0.440 \mathrm{mmol})$ was suspended in $\mathrm{CH}_{2} \mathrm{Cl}_{2}\left(10 \mathrm{~cm}^{3}\right)$, and $1,4-$ bis(diphenylphosphino)benzene (102 $\mathrm{mg}, 0.229 \mathrm{mmol}$ ) was added. 
The reaction mixture was stirred for $30 \mathrm{~min}$. The yellow transparent solution was diluted with toluene $\left(5 \mathrm{~cm}^{3}\right)$, concentrated to a volume of ca. $7 \mathrm{~cm}^{3}$. The yellow solid was separated by centrifugation, washed with toluene $\left(5 \mathrm{~cm}^{3}\right)$ and diethyl ether $\left(3 \times 5 \mathrm{~cm}^{3}\right)$, and vaccuum-dried (236 mg, 95\%). An analytically pure sample was obtained by recrystallization from $\mathrm{CH}_{2} \mathrm{Cl}_{2}$ /diethyl ether at $+5{ }^{\circ} \mathrm{C}$. ${ }^{31} \mathrm{P}\left\{{ }^{1} \mathrm{H}\right\} \mathrm{NMR}\left(\mathrm{CDCl}_{3} ; \delta\right): 42.0(\mathrm{~s}) .{ }^{1} \mathrm{H} \mathrm{NMR}\left(\mathrm{CDCl}_{3} ; \delta\right): 7.63-7.46$ (m, 24H), $7.41\left(\mathrm{dm}, \mathrm{C}_{6} \mathrm{H}_{4}-\mathrm{N}\left(\mathrm{CH}_{3}\right)_{2}, J(\mathrm{H}-\mathrm{H}) 9.0 \mathrm{~Hz}, 4 \mathrm{H}\right), 6.61$ $\left(\mathrm{dm}, \mathrm{C}_{6} \mathrm{H}_{4}-\mathrm{N}\left(\mathrm{CH}_{3}\right)_{2}, J(\mathrm{H}-\mathrm{H}) 9.0 \mathrm{~Hz}, 4 \mathrm{H}\right), 2.96\left(\mathrm{~s}, \mathrm{~N}\left(\mathrm{CH}_{3}\right)_{2}, 12 \mathrm{H}\right)$. Anal. calcd for $\mathrm{C}_{50} \mathrm{H}_{44} \mathrm{Au}_{2} \mathrm{~N}_{2} \mathrm{P}_{2}$ : C, 53.20; H, 3.93; N, 2.48. Found: C, 53.01; H, 3.95; N, 2.40 .

$\left(\mathrm{AuC}_{2} \mathrm{C}_{6} \mathrm{H}_{4} \mathrm{NO}_{2}\right)_{2} \mathrm{P}\left(\mathrm{NC}_{4} \mathrm{H}_{4}\right)_{2} \mathrm{C}_{6} \mathrm{H}_{4} \mathbf{P}\left(\mathrm{NC}_{4} \mathrm{H}_{4}\right)_{2}$ (5). Complex 5 was generated analogously to $\mathbf{3}$, using $\left(\mathrm{AuC}_{2} \mathrm{C}_{6} \mathrm{H}_{4} \mathrm{NO}_{2}\right)_{n}(150 \mathrm{mg}$, $0.437 \mathrm{mmol}$ ) and 1,4-bis(dipyrrolylphosphino)benzene (92 mg, $0.229 \mathrm{mmol}$ ). Yield: $202 \mathrm{mg}, 85 \%$. An analytically pure sample was obtained by recrystallization from $\mathrm{CH}_{2} \mathrm{Cl}_{2}$ /diethyl ether at +5 ${ }^{\circ} \mathrm{C} .{ }^{31} \mathrm{P}\left\{{ }^{1} \mathrm{H}\right\}$ NMR $\left(\mathrm{CDCl}_{3} ; \delta\right): 95.0$ (s, br). ${ }^{1} \mathrm{H} \mathrm{NMR}\left(\mathrm{CDCl}_{3} ; \delta\right)$ : $8.15\left(\mathrm{dm}, \mathrm{C}_{6} \mathrm{H}_{4}-\mathrm{NO}_{2}, J(\mathrm{H}-\mathrm{H}) 8.9 \mathrm{~Hz}, 4 \mathrm{H}\right), 7.59\left(\mathrm{dm}, \mathrm{C}_{6} \mathrm{H}_{4}-\mathrm{NO}_{2}\right.$, $J(\mathrm{H}-\mathrm{H}) 8.9 \mathrm{~Hz}, 4 \mathrm{H}), 7.38\left(\mathrm{~m}, \mathrm{P}-\mathrm{C}_{6} \mathrm{H}_{4}-\mathrm{P}, 4 \mathrm{H}\right), 7.10\left(\mathrm{~m}, \mathrm{NC}_{4} \mathrm{H}_{4}\right.$, $8 \mathrm{H}), 6.52\left(\mathrm{dm}, \mathrm{NC}_{4} \mathrm{H}_{4}, J(\mathrm{H}-\mathrm{H}) 1.8 \mathrm{~Hz}, 8 \mathrm{H}\right)$. Anal. calcd for $\mathrm{C}_{38} \mathrm{H}_{28} \mathrm{Au}_{2} \mathrm{~N}_{6} \mathrm{O}_{4} \mathrm{P}_{2}$ : C, 41.93; H, 2.59; N, 7.72. Found: C, 42.23; H, 2.59; N, 7.39.

$\left(\mathrm{AuC}_{2} \mathbf{P h}\right)_{\mathbf{2}} \mathbf{P}\left(\mathrm{NC}_{\mathbf{4}} \mathbf{H}_{\mathbf{4}}\right)_{2} \mathbf{C}_{\mathbf{6}} \mathbf{H}_{\mathbf{4}} \mathbf{P}\left(\mathrm{NC}_{\mathbf{4}} \mathrm{H}_{4}\right)_{\mathbf{2}} \quad$ (6). $\left(\mathrm{AuC}_{2} \mathrm{C}_{6} \mathrm{H}_{5}\right)_{n}$ $\mathrm{mg}, 0.503 \mathrm{mmol})$ was suspended in $\mathrm{CH}_{2} \mathrm{Cl}_{2}\left(10 \mathrm{~cm}^{3}\right)$, and 1,4bis(dipyrrolylphosphino)benzene (106 mg, $0.264 \mathrm{mmol}$ ) was added. The reaction mixture was stirred for $30 \mathrm{~min}$. The resulting suspension was evaporated, dissolved in $\mathrm{CHCl}_{3}$ (ca. $35 \mathrm{~cm}^{3}$ ), diluted with toluene $\left(5 \mathrm{~cm}^{3}\right)$, and treated as $\mathbf{2}$. A pale yellowish solid was formed, $230 \mathrm{mg}, 92 \%$. An analytically pure sample was obtained by recrystallization from $\mathrm{CH}_{2} \mathrm{Cl}_{2} /$ diethyl ether at $+5{ }^{\circ} \mathrm{C} .{ }^{31} \mathrm{P}\left\{{ }^{1} \mathrm{H}\right\}$ NMR $\left(\mathrm{CDCl}_{3} ; \delta\right): 95.5$ (s, br). ${ }^{1} \mathrm{H} \mathrm{NMR}\left(\mathrm{CDCl}_{3} ; \delta\right): 7.50(\mathrm{~m}, 4 \mathrm{H})$, $7.37-7.23(\mathrm{~m}, 10 \mathrm{H}), 7.11\left(\mathrm{~m}, \mathrm{NC}_{4} \mathrm{H}_{4}, 8 \mathrm{H}\right), 6.50\left(\mathrm{dm}, \mathrm{NC}_{4} \mathrm{H}_{4}\right.$, $J(\mathrm{H}-\mathrm{H}) 2.1 \mathrm{~Hz}, 8 \mathrm{H})$. Anal. calcd for $\mathrm{C}_{38} \mathrm{H}_{30} \mathrm{Au}_{2} \mathrm{~N}_{4} \mathrm{P}_{2}: \mathrm{C}, 45.71 ; \mathrm{H}$, 3.03; N, 5.61. Found: C, 45.94; H, 3.08; N, 5.50.

$\left(\mathrm{AuC}_{2} \mathrm{C}_{6} \mathrm{H}_{4} \mathrm{OMe}\right)_{2} \mathbf{P}\left(\mathrm{NC}_{4} \mathrm{H}_{4}\right)_{2} \mathrm{C}_{6} \mathrm{H}_{4} \mathbf{P}\left(\mathrm{NC}_{4} \mathrm{H}_{4}\right)_{2}(7)$. $\left(\mathrm{AuC}_{2} \mathrm{C}_{6} \mathrm{H}_{4}-\right.$ $\mathrm{OMe})_{n}(150 \mathrm{mg}, 0.457 \mathrm{mmol})$ was suspended in $\mathrm{CH}_{2} \mathrm{Cl}_{2}\left(10 \mathrm{~cm}^{3}\right)$, and 1,4-bis(dipyrrolylphosphino)benzene ( $95 \mathrm{mg}, 0.236 \mathrm{mmol}$ ) was added. The reaction mixture was stirred for $30 \mathrm{~min}$. The white suspension was diluted with toluene $\left(10 \mathrm{~cm}^{3}\right)$, and the solid was separated by centrifugation, washed with toluene $\left(5 \mathrm{~cm}^{3}\right)$ and diethyl ether, and dried under a vacuum. The product (220 mg, 91\%) was obtained as a white powder. An analytically pure sample was obtained by extraction with chloroform passing through a layer of neutral $\mathrm{Al}_{2} \mathrm{O}_{3}(1 \times 4 \mathrm{~cm})$, evaporation, and washing of the residue with diethyl ether. ${ }^{31} \mathrm{P}\left\{{ }^{1} \mathrm{H}\right\}$ NMR $\left(\mathrm{CDCl}_{3} ; \delta\right)$ : 95.7 (s, br). ${ }^{1} \mathrm{H}$ NMR $\left(\mathrm{CDCl}_{3} ; \delta\right): 7.45\left(\mathrm{dm}, \mathrm{C}_{6} \mathrm{H}_{4}-\mathrm{OCH}_{3}, J(\mathrm{H}-\mathrm{H}) 8.8 \mathrm{~Hz}, 4 \mathrm{H}\right), 7.32$ $\left(\mathrm{m}, \mathrm{P}-\mathrm{C}_{6} \mathrm{H}_{4}-\mathrm{P}, 4 \mathrm{H}\right), 7.10\left(\mathrm{~m}, \mathrm{NC}_{4} \mathrm{H}_{4}, 8 \mathrm{H}\right), 6.83\left(\mathrm{dm}, \mathrm{C}_{6} \mathrm{H}_{4}-\mathrm{OCH}_{3}\right.$, $J(\mathrm{H}-\mathrm{H}) 8.8 \mathrm{~Hz}, 4 \mathrm{H}), 6.49\left(\mathrm{~m}, \mathrm{NC}_{4} \mathrm{H}_{4}, 8 \mathrm{H}\right), 3.81\left(\mathrm{~s}, \mathrm{OCH}_{3}, 6 \mathrm{H}\right)$. Anal. calcd for $\mathrm{C}_{40} \mathrm{H}_{34} \mathrm{Au}_{2} \mathrm{~N}_{4} \mathrm{O}_{2} \mathrm{P}_{2}$ : C, 45.38; H, 3.24; N, 5.29. Found: C, 45.53; H, 3.26; N, 5.24.

General Procedure for the Preparation of Heterometallic Au-Cu Complexes. Complexes 2-7 (0.100 mmol) were suspended in $\mathrm{CH}_{2} \mathrm{Cl}_{2}\left(8 \mathrm{~cm}^{3}\right)$ and diluted with diethyl ether $\left(4 \mathrm{~cm}^{3}\right)$, and a solution of $\mathrm{Cu}(\mathrm{NCMe})_{4} \mathrm{PF}_{6}(0.067 \mathrm{mmol})$ in $\mathrm{CH}_{2} \mathrm{Cl}_{2}(3$ $\mathrm{cm}^{3}$ ) was added dropwise to afford a transparent orange to red solution (except 12, orange suspension formed). The solvent was removed on a rotovap (12 was separated from the pale solution by centrifugation). The solid residue was dissolved in acetone $\left(3 \mathrm{~cm}^{3}\right)$, filtered, and precipitated by the addition of hexane or diethyl ether to give a microcrystalline solid. If necessary, the samples were recrystallized by the gas-phase diffusion of pentane or diethyl ether into their acetone solutions at $+5{ }^{\circ} \mathrm{C}$. Complex $\left[\left\{\mathrm{Au}_{3} \mathrm{Cu}_{2}\left(\mathrm{C}_{2} \mathrm{Ph}\right)_{6}\right\} \mathrm{Au}_{3}\left(\mathrm{PPh}_{2} \mathrm{C}_{6} \mathrm{H}_{4} \mathrm{PPh}_{2}\right)_{3}\right]\left[\mathrm{PF}_{6}\right]_{2}$ (9) was reported earlier. $^{6}$

$\left[\left\{\mathrm{Au}_{3} \mathrm{Cu}_{2}\left(\mathrm{C}_{2} \mathrm{C}_{6} \mathrm{H}_{4} \mathrm{NO}_{2}\right)_{6}\right\} \mathrm{Au}_{3}\left(\mathrm{PPh}_{2} \mathrm{C}_{6} \mathrm{H}_{4} \mathrm{PPh}_{2}\right)_{3}\right]\left[\mathrm{PF}_{6}\right]_{2}(8) . \mathrm{An}$ orange compound was formed. Yield: $82 \%$. ES MS $(\mathrm{m} / \mathrm{z})$ : $\left[\mathrm{Au}_{6} \mathrm{Cu}_{2}\left(\mathrm{C}_{2} \mathrm{C}_{6} \mathrm{H}_{4} \mathrm{NO}_{2}\right)_{6}\left(\mathrm{PPh}_{2} \mathrm{C}_{6} \mathrm{H}_{4} \mathrm{PPh}_{2}\right)_{3}\right]^{2+}, 1762$ (calcd, 1762). ${ }^{31} \mathrm{P}\left\{{ }^{1} \mathrm{H}\right\}$ NMR (acetone- $\mathrm{d}_{6} ; \delta$ ): 44.7 (s, 3P), -144.8 (sept, 1P, $\mathrm{PF}_{6}$ ). ${ }^{1} \mathrm{H}$ NMR (acetone- $\mathrm{d}_{6} ; \delta$ ), $\left\{\mathrm{Au}\left(\mathrm{C}_{2} \mathrm{C}_{6} \mathrm{H}_{4} \mathrm{NO}_{2}\right)_{2}\right\}: 7.65$ (d, H-meta, 12H, $J(\mathrm{H}-\mathrm{H}) 8.8 \mathrm{~Hz}$ ), 7.11 (dd, H-ortho, 12H, $J(\mathrm{H}-\mathrm{H}) 8.8 \mathrm{~Hz}$ ). ${ }^{1} \mathrm{H}$ NMR (acetone- $\left.\mathrm{d}_{6} ; \delta\right)$, diphosphine: $8.02\left(\mathrm{dm}\left(\mathrm{AXX}^{\prime}\right), \mathrm{H}\right.$-ortho, $24 \mathrm{H}, J(\mathrm{H}-\mathrm{H}) 6.7, J(\mathrm{P}-\mathrm{H}) 13.6 \mathrm{~Hz}), 7.91\left(\mathrm{~m}\left(\mathrm{~A}_{2} \mathrm{X}_{2}\right),\left\{\mathrm{P}-\mathrm{C}_{6} \mathrm{H}_{4}-\mathrm{P}\right\}\right.$ $12 \mathrm{H},<J(\mathrm{P}-\mathrm{H})>3.4 \mathrm{~Hz}), 7.68(\mathrm{t}, \mathrm{H}-\mathrm{para}, 12 \mathrm{H}, J(\mathrm{H}-\mathrm{H}) 7.5 \mathrm{~Hz})$, 7.51 (dd, H-meta, 24H, J(H-H) 6.7, 7.5 Hz). ${ }^{13} \mathrm{C}$ NMR (acetone$\left.\mathrm{d}_{6} ; \delta\right),\left\{\mathrm{Au}\left(\mathrm{C}_{2} \mathrm{C}_{6} \mathrm{H}_{4} \mathrm{NO}_{2}\right)_{2}\right\}: 148.33$ (s, C-para- $\left.\mathrm{NO}_{2}\right), 134.47$ and 124.78 (s, C-meta and C-ortho), 123.52 (s, C-ipso), 112.86 (s, $\mathrm{C} \equiv \mathrm{C}-\mathrm{Au}), 117.39$ (s, $\mathrm{C} \equiv \mathrm{C}-\mathrm{Au}) .{ }^{13} \mathrm{C}$ NMR (acetone- $\left.\mathrm{d}_{6} ; \delta\right)$, diphosphine: $137.12\left(\mathrm{~m}(\mathrm{AXX}), \mathrm{C}\left(-\mathrm{C}_{6} \mathrm{H}_{4}-\right), J(\mathrm{P}-\mathrm{C}) 59 \mathrm{~Hz}\right)$, 136.92 (m (AXX'), C-ortho, $J(\mathrm{P}-\mathrm{C}) 16.5 \mathrm{~Hz}), 135.73\left(\mathrm{~m}\left(\mathrm{AXX}^{\prime}\right)\right.$, $\mathrm{C}(\mathrm{H})\left(-\mathrm{C}_{6} \mathrm{H}_{4}-\right), J(\mathrm{P}-\mathrm{C})$ ca. $\left.12 \mathrm{~Hz}\right), 134.63$ (s, C-para), 131.61 (m (AXX'), C-meta, $J(\mathrm{P}-\mathrm{C}) 11.9 \mathrm{~Hz}), 129.50$ (m (AXX'), C-ipso, $J(\mathrm{P}-\mathrm{C}) 56.7 \mathrm{~Hz})$. Anal. calcd for $\mathrm{C}_{138} \mathrm{H}_{96} \mathrm{Au}_{6} \mathrm{Cu}_{2} \mathrm{~F}_{12} \mathrm{~N}_{6} \mathrm{O}_{12} \mathrm{P}_{8}: \mathrm{C}$, 43.45; H, 2.54; N, 2.20. Found: C, 43.34; H, 2.87; N, 2.16.

$\left[\left\{\mathrm{Au}_{3} \mathrm{Cu}_{2}\left(\mathrm{C}_{2} \mathrm{C}_{6} \mathrm{H}_{4} \mathrm{OMe}\right)_{6}\right\} \mathrm{Au}_{3}\left(\mathrm{PPh}_{2} \mathrm{C}_{6} \mathrm{H}_{4} \mathrm{PPh}_{2}\right)_{3}\right]\left[\mathrm{PF}_{6}\right]_{2}(10)$. An orange-red compound was formed. Yield: $86 \%$. ES MS $(\mathrm{m} / \mathrm{z})$ : $\left[\mathrm{Au}_{6} \mathrm{Cu}_{2}\left(\mathrm{C}_{2} \mathrm{C}_{6} \mathrm{H}_{4} \mathrm{OMe}\right)_{6}\left(\mathrm{PPh}_{2} \mathrm{C}_{6} \mathrm{H}_{4} \mathrm{PPh}_{2}\right)_{3}\right]^{2+}, 1717$ (calcd, 1717). ${ }^{31} \mathrm{P}\left\{{ }^{1} \mathrm{H}\right\}$ NMR (acetone- $\mathrm{d}_{6} ; \delta$ ): 43.4 (s, 3P), -144.8 (sept, 1P, $\mathrm{PF}_{6}$ ). ${ }^{1} \mathrm{H}$ NMR (acetone- $\mathrm{d}_{6} ; \delta$ ), $\left\{\mathrm{Au}\left(\mathrm{C}_{2} \mathrm{C}_{6} \mathrm{H}_{4} \mathrm{NO}_{2}\right)_{2}\right\}: 6.69$ (d, H-ortho, $12 \mathrm{H}, J(\mathrm{H}-\mathrm{H}) 8.7 \mathrm{~Hz}), 6.39$ (dd, H-meta, 12H, $J(\mathrm{H}-\mathrm{H}) 8.7 \mathrm{~Hz}$ ), 3.72 (s, 18H, OMe). ${ }^{1} \mathrm{H}$ NMR (acetone- $\mathrm{d}_{6} ; \delta$ ), diphosphine: 7.97 (dm(AXX'), H-ortho, 24H, $J(\mathrm{H}-\mathrm{H}) 6.7, J(\mathrm{P}-\mathrm{H}) 7.3 \mathrm{~Hz}), 7.75$ $\left(\mathrm{m}\left(\mathrm{A}_{2} \mathrm{X}_{2}\right),\left\{\mathrm{P}-\mathrm{C}_{6} \mathrm{H}_{4}-\mathrm{P}\right\} 12 \mathrm{H},<J(\mathrm{P}-\mathrm{H})>3.4 \mathrm{~Hz}\right), 7.66(\mathrm{t}, \mathrm{H}-\mathrm{para}$, 12H, $J(\mathrm{H}-\mathrm{H}) 7.5 \mathrm{~Hz}), 7.48(\mathrm{dd}, \mathrm{H}-$ meta, 24H, $J(\mathrm{H}-\mathrm{H}) 6.7,7.5$ $\mathrm{Hz}$ ). ${ }^{13} \mathrm{C}$ NMR (acetone- $\left.\mathrm{d}_{6} ; \delta\right),\left\{\mathrm{Au}\left(\mathrm{C}_{2} \mathrm{C}_{6} \mathrm{H}_{4} \mathrm{NO}_{2}\right)_{2}\right\}: 161.24(\mathrm{~s}$, C-para-OMe), 135.07 and 115.07 (s, C-meta and C-ortho), 117.67 (s, C-ipso), 114.43 (s, $\underline{C} \equiv \mathrm{C}-\mathrm{Au}$ ), 110.94 (s, C $\equiv \mathrm{C}-\mathrm{Au}), 56.35$ (s, OMe). ${ }^{13} \mathrm{C}$ NMR (acetone- $\left.\mathrm{d}_{6} ; \delta\right)$, diphosphine: $137.43\left(\mathrm{~m}\left(\mathrm{AXX}^{\prime}\right)\right.$, $\left.\mathrm{C}\left(-\mathrm{C}_{6} \mathrm{H}_{4}-\right), J(\mathrm{P}-\mathrm{C}) 59 \mathrm{~Hz}\right), 137.03\left(\mathrm{~m}\left(\mathrm{AXX}^{\prime}\right), \mathrm{C}\right.$-ortho, $J(\mathrm{P}-\mathrm{C})$ $16.4 \mathrm{~Hz}), 135.61\left(\mathrm{~m}\left(\mathrm{AXX}^{\prime}\right), \mathrm{C}(\mathrm{H})\left(-\mathrm{C}_{6} \mathrm{H}_{4}-\right), J(\mathrm{P}-\mathrm{C})\right.$ ca. $\left.11 \mathrm{~Hz}\right)$, 134.55 (s, C-para), 131.61 (m (AXX'), C-meta, $J(\mathrm{P}-\mathrm{C}) 11.8 \mathrm{~Hz})$, $130.01\left(\mathrm{~m}\left(\mathrm{AXX}^{\prime}\right), \mathrm{C}\right.$-ipso, $\left.J(\mathrm{P}-\mathrm{C}) 55.2 \mathrm{~Hz}\right)$. Anal. calcd for $\mathrm{C}_{144} \mathrm{H}_{114} \mathrm{Au}_{6} \mathrm{Cu}_{2} \mathrm{~F}_{12} \mathrm{O}_{6} \mathrm{P}_{8}: \mathrm{C}, 46.43 ; \mathrm{H}, 3.08$. Found: $\mathrm{C}, 46.51 ; \mathrm{H}$, 3.15 .

$\left[\left\{\mathrm{Au}_{3} \mathrm{Cu}_{2}\left(\mathrm{C}_{2} \mathrm{C}_{6} \mathrm{H}_{4} \mathrm{NMe}_{2}\right)_{6}\right\} \mathrm{Au}_{3}\left(\mathrm{PPh}_{2} \mathrm{C}_{6} \mathrm{H}_{4} \mathrm{PPh}_{2}\right)_{3}\right]\left[\mathrm{PF}_{6}\right]_{2}$ (11). A very dark red compound was formed. Yield: 71\%. ES MS $(\mathrm{m} / \mathrm{z})$ : $\left[\mathrm{Au}_{6} \mathrm{Cu}_{2}\left(\mathrm{C}_{2} \mathrm{C}_{6} \mathrm{H}_{4} \mathrm{NMe}_{2}\right)_{6}\left(\mathrm{PPh}_{2} \mathrm{C}_{6} \mathrm{H}_{4} \mathrm{PPh}_{2}\right)_{3}\right]^{2+}, 1756$ (calcd, 1756). ${ }^{31} \mathrm{P}\left\{{ }^{1} \mathrm{H}\right\}$ NMR (acetone- $\mathrm{d}_{6} ; \delta$ ): 42.9 (s, 3P), -144.8 (sept, 1P, $\mathrm{PF}_{6}$ ). ${ }^{1} \mathrm{H}$ NMR (acetone- $\mathrm{d}_{6} ; \delta$ ), $\left\{\mathrm{Au}\left(\mathrm{C}_{2} \mathrm{C}_{6} \mathrm{H}_{4} \mathrm{NMe}_{2}\right)_{2}\right\}: 6.58$ (d, H-ortho, $12 \mathrm{H}, J(\mathrm{H}-\mathrm{H}) 8.8 \mathrm{~Hz}), 6.39$ (dd, H-meta, 12H, $J(\mathrm{H}-\mathrm{H}) 8.8 \mathrm{~Hz}$ ), $2.90\left(\mathrm{~s}, 36 \mathrm{H}, \mathrm{NMe}_{2}\right) .{ }^{1} \mathrm{H}$ NMR (acetone- $\mathrm{d}_{6} ; \delta$ ), diphosphine: 7.98 (dm(AXX'), H-ortho, 24H, J(H-H) 6.7, J(P-H) $6.7 \mathrm{~Hz}), 7.73$ $\left(\mathrm{m}\left(\mathrm{A}_{2} \mathrm{X}_{2}\right),\left\{\mathrm{P}-\mathrm{C}_{6} \mathrm{H}_{4}-\mathrm{P}\right\} 12 \mathrm{H},<J(\mathrm{P}-\mathrm{H})>3.3 \mathrm{~Hz}\right), 7.64$ (t, H-para, $12 \mathrm{H}, J(\mathrm{H}-\mathrm{H}) 7.5 \mathrm{~Hz}), 7.45(\mathrm{dd}, \mathrm{H}-m e t a, 24 \mathrm{H}, J(\mathrm{H}-\mathrm{H}) 6.7,7.5$ $\mathrm{Hz}$ ). ${ }^{13} \mathrm{C}$ NMR (acetone- $\mathrm{d}_{6} ; \delta$ ), $\left\{\mathrm{Au}\left(\mathrm{C}_{2} \mathrm{C}_{6} \mathrm{H}_{4} \mathrm{NMe}_{2}\right)_{2}\right\}: 151.50$ (s, C-para-NMe ${ }_{2}$ ), 135.10 and 113.29 (s, C-meta and C-ortho), 112.91

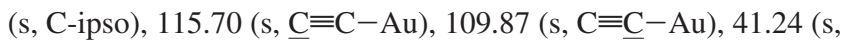
$\left.\mathrm{NMe}_{2}\right) .{ }^{13} \mathrm{C}$ NMR (acetone-d $\left.\mathrm{d}_{6} ; \delta\right)$, diphosphine: $137.50\left(\mathrm{~m}\left(\mathrm{AXX}^{\prime}\right)\right.$, $\left.\mathrm{C}\left(-\mathrm{C}_{6} \mathrm{H}_{4}-\right), J(\mathrm{P}-\mathrm{C}) 59 \mathrm{~Hz}\right), 136.99\left(\mathrm{~m}\left(\mathrm{AXX}^{\prime}\right), \mathrm{C}\right.$-ortho, $J(\mathrm{P}-\mathrm{C})$ $16.4 \mathrm{~Hz}), 135.51\left(\mathrm{~m}\left(\mathrm{AXX}^{\prime}\right), \mathrm{C}(\mathrm{H})\left(-\mathrm{C}_{6} \mathrm{H}_{4}-\right), J(\mathrm{P}-\mathrm{C})\right.$ ca. $\left.11 \mathrm{~Hz}\right)$, 134.36 (s, C-para), 131.62 (m (AXX'), C-meta, $J(\mathrm{P}-\mathrm{C}) 11.7 \mathrm{~Hz})$, 130.21 (m $\left(\mathrm{AXX}^{\prime}\right), \mathrm{C}$-ipso, $\left.J(\mathrm{P}-\mathrm{C}) 54.5 \mathrm{~Hz}\right)$. Anal. calcd for $\mathrm{C}_{150} \mathrm{H}_{132} \mathrm{Au}_{6} \mathrm{Cu}_{2} \mathrm{~F}_{12} \mathrm{~N}_{6} \mathrm{P}_{8}: \mathrm{C}, 47.37 ; \mathrm{H}, 3.50 ; \mathrm{N}, 2.21$. Found: $\mathrm{C}$, 47.53; H, 3.46; N, 2.13. 
$\left[\left\{\mathrm{Au}_{3} \mathrm{Cu}_{2}\left(\mathrm{C}_{2} \mathrm{C}_{6} \mathrm{H}_{4} \mathrm{NO}_{2}\right)_{6}\right\} \mathrm{Au}_{3}\left(\mathrm{P}\left(\mathrm{NC}_{4} \mathrm{H}_{4}\right)_{2} \mathrm{C}_{6} \mathrm{H}_{4} \mathrm{P}\left(\mathrm{NC}_{4} \mathrm{H}_{4}\right)_{2}\right)_{3}\right]-$ $\left[\mathrm{PF}_{\mathbf{6}}\right]_{2} \mathbf{( 1 2 )}$. An orange compound was formed. Yield: $84 \%$. ES

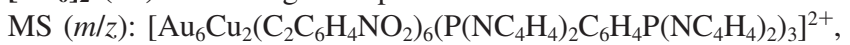
1696 (calcd, 1696). ${ }^{31} \mathrm{P}\left\{{ }^{1} \mathrm{H}\right\}$ NMR (acetone- $\mathrm{d}_{6} ; \delta$ ): 89.6 (s, 3P), -144.8 (sept, 1P, $\quad \mathrm{PF}_{6}$ ). ${ }^{1} \mathrm{H} \quad \mathrm{NMR}$ (acetone- $\mathrm{d}_{6} ; \quad \delta$ ), $\left\{\mathrm{Au}\left(\mathrm{C}_{2} \mathrm{C}_{6} \mathrm{H}_{4} \mathrm{NO}_{2}\right)_{2}\right\}$ : H-ortho $7.68(\mathrm{~d}, 12 \mathrm{H}, J(\mathrm{H}-\mathrm{H}) 8.8 \mathrm{~Hz})$, H-meta $7.22(\mathrm{~d}, 12 \mathrm{H}, J(\mathrm{H}-\mathrm{H}) 8.8 \mathrm{~Hz}) .{ }^{1} \mathrm{H}$ NMR (acetone- $\left.\mathrm{d}_{6} ; \delta\right)$, diphos-

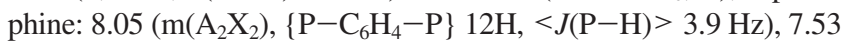
(dm(AXX'), Pyr 2-CH, 24H, $J(\mathrm{H}-\mathrm{H}) 2.1, J(\mathrm{P}-\mathrm{H}) 4.2 \mathrm{~Hz}), 6.49$ (dm(AXX'), Pyr 3-CH, 24H, J(H-H) 2.1, J(P-H) $2.0 \mathrm{~Hz}) .{ }^{13} \mathrm{C}$ NMR (acetone- $\left.\mathrm{d}_{6} ; \delta\right),\left\{\mathrm{Au}\left(\mathrm{C}_{2} \mathrm{C}_{6} \mathrm{H}_{4} \mathrm{NO}_{2}\right)_{2}\right\}: 149.97\left(\mathrm{~s}, \mathrm{C}-\right.$ para- $\left.\mathrm{NO}_{2}\right)$, 135.09 and 124.95 (s, C-ortho and C-meta), 130.31 (s, C-ipso), $113.90(\mathrm{~s}, \underline{\mathrm{C}} \equiv \mathrm{C}-\mathrm{Au}), 116.48(\mathrm{~s}, \mathrm{C} \equiv \mathrm{C}-\mathrm{Au}) .{ }^{13} \mathrm{C}$ NMR (acetone$\left.\mathrm{d}_{6} ; \delta\right)$, diphosphine: $137.69\left(\mathrm{~m}\left(\mathrm{AXX}^{\prime}\right), \mathrm{C}\left(-\mathrm{C}_{6} \mathrm{H}_{4}-\right), J(\mathrm{P}-\mathrm{C}) 82.6\right.$ $\mathrm{Hz}), 135.74\left(\mathrm{~m}\left(\mathrm{AXX}^{\prime}\right), \mathrm{C}(\mathrm{H})\left(-\mathrm{C}_{6} \mathrm{H}_{4}-\right), J(\mathrm{P}-\mathrm{C})\right.$ ca. $\left.14 \mathrm{~Hz}\right)$, 127.78 (m (AXX'), Pyr 2-C, $J(\mathrm{P}-\mathrm{C})$ ca. $10 \mathrm{~Hz}), 117.63$ (s, Pyr 3-C). Anal. calcd for $\mathrm{C}_{114} \mathrm{H}_{84} \mathrm{Au}_{6} \mathrm{Cu}_{2} \mathrm{~F}_{12} \mathrm{~N}_{18} \mathrm{O}_{12} \mathrm{P}_{8}$ : C, 37.18; H, 2.30; N, 6.85. Found: C, 37.16; H, 2.35; N, 6.57.

$\left[\left\{\mathrm{Au}_{3} \mathrm{Cu}_{2}\left(\mathrm{C}_{2} \mathrm{Ph}\right)_{6}\right\} \mathrm{Au}_{3}\left(\mathrm{P}\left(\mathrm{NC}_{4} \mathrm{H}_{4}\right)_{2} \mathrm{C}_{6} \mathrm{H}_{4} \mathbf{P}\left(\mathrm{NC}_{4} \mathrm{H}_{4}\right)_{2}\right)_{3}\right]\left[\mathrm{PF}_{6}\right]_{2}(13)$. An orange-red compound was formed. Yield: $91 \%$. ES MS $(\mathrm{m} / \mathrm{z})$ :

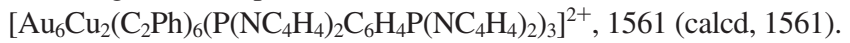
${ }^{31} \mathrm{P}\left\{{ }^{1} \mathrm{H}\right\}$ NMR (acetone- $\mathrm{d}_{6} ; \delta$ ): 89.8 (s, 3P), -144.8 (sept, $1 \mathrm{P}, \mathrm{PF}_{6}$ ). ${ }^{1} \mathrm{H}$ NMR (acetone- $\mathrm{d}_{6} ; \delta$ ), $\left\{\mathrm{Au}\left(\mathrm{C}_{2} \mathrm{Ph}\right)_{2}\right\}: 7.13$ (tt, H-para, $6 \mathrm{H}$, $J(\mathrm{H}-\mathrm{H}) 7.3,1.5 \mathrm{~Hz})$. ABX system, H-ortho-H-meta: $6.89(\mathrm{dd}, 12 \mathrm{H}$, $J(\mathrm{H}-\mathrm{H}) 6.8,1.5 \mathrm{~Hz}), 6.85(\mathrm{dd}, 12 \mathrm{H}, J(\mathrm{H}-\mathrm{H}) 6.8,7.3 \mathrm{~Hz}) .{ }^{1} \mathrm{H} \mathrm{NMR}$ (acetone- $\left.\mathrm{d}_{6} ; \delta\right)$, diphosphine: $7.51\left(\mathrm{dm}\left(\mathrm{AXX}^{\prime}\right)\right.$, Pyr 2-CH, $24 \mathrm{H}$,

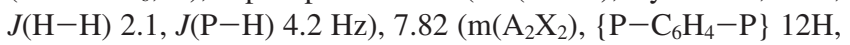
$<J(\mathrm{P}-\mathrm{H})>3.9 \mathrm{~Hz}), 6.48\left(\mathrm{dm}\left(\mathrm{AXX}^{\prime}\right), \mathrm{Pyr} 3-\mathrm{CH}, 24 \mathrm{H}, J(\mathrm{H}-\mathrm{H})\right.$ 2.1, J(P-H) $2.0 \mathrm{~Hz}) .{ }^{13} \mathrm{C}$ NMR (acetone- $\left.\mathrm{d}_{6} ; \delta\right),\left\{\mathrm{Au}\left(\mathrm{C}_{2} \mathrm{Ph}\right)_{2}\right\}$ : 134.00 and 129.85 (s, C-ortho and C-meta), 130.19 (s, C-para),

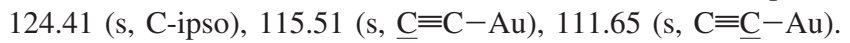
${ }^{13} \mathrm{C}$ NMR (acetone- $\mathrm{d}_{6} ; \delta$ ), diphosphine: 137.87 (m $\left(\mathrm{AXX} \mathrm{X}^{\prime}\right), \mathrm{C}$ $\left.\left(-\mathrm{C}_{6} \mathrm{H}_{4}-\right), J(\mathrm{P}-\mathrm{C}) 82.5 \mathrm{~Hz}\right), 135.21\left(\mathrm{~m}(\mathrm{AXX}), \mathrm{C}(\mathrm{H})\left(-\mathrm{C}_{6} \mathrm{H}_{4}-\right)\right.$, $J(\mathrm{P}-\mathrm{C})$ ca. $13 \mathrm{~Hz}), 127.72\left(\mathrm{~m}\left(\mathrm{AXX}^{\prime}\right), \operatorname{Pyr} 2-\mathrm{C}, J(\mathrm{P}-\mathrm{C})\right.$ ca. $\left.9 \mathrm{~Hz}\right)$, 117.38 (s, Pyr 3-C). Anal. calcd for $\mathrm{C}_{114} \mathrm{H}_{90} \mathrm{Au}_{6} \mathrm{Cu}_{2} \mathrm{~F}_{12} \mathrm{~N}_{12} \mathrm{P}_{8}$ : C, 40.12; H, 2.66; N, 4.93. Found: C, 39.73; H, 2.61; N, 4.77.

$\left[\left\{\mathrm{Au}_{3} \mathrm{Cu}_{2}\left(\mathrm{C}_{2} \mathrm{C}_{6} \mathrm{H}_{4} \mathrm{OMe}\right)_{6}\right\} \mathrm{Au}_{3}\left(\mathbf{P}\left(\mathrm{NC}_{4} \mathrm{H}_{4}\right)_{2} \mathrm{C}_{6} \mathrm{H}_{4} \mathrm{P}\left(\mathrm{NC}_{4} \mathrm{H}_{4}\right)_{2}\right)_{3}\right]-$ $\left[\mathrm{PF}_{\mathbf{6}}\right]_{2} \mathbf{( 1 4 )}$. A deep red complex was formed. Yield: 78\%. ES MS

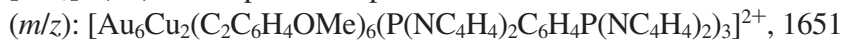
(calcd, 1651). ${ }^{31} \mathrm{P}\left\{{ }^{1} \mathrm{H}\right\}$ NMR (acetone- $\mathrm{d}_{6} ; \delta$ ): 90.0 (s, 3P), -144.8 (sept, $\left.1 \mathrm{P}, \mathrm{PF}_{6}\right) .{ }^{1} \mathrm{H}$ NMR (acetone- $\mathrm{d}_{6} ; \delta$ ), $\left\{\mathrm{Au}\left(\mathrm{C}_{2} \mathrm{C}_{6} \mathrm{H}_{4} \mathrm{OMe}\right)_{2}\right\}$ : H-ortho $6.74(\mathrm{~d}, 12 \mathrm{H}, J(\mathrm{H}-\mathrm{H}) 8.8 \mathrm{~Hz}), \mathrm{H}$-meta $6.41(\mathrm{~d}, 12 \mathrm{H}$, $J(\mathrm{H}-\mathrm{H}) 8.8 \mathrm{~Hz}) .{ }^{1} \mathrm{H}$ NMR (acetone- $\mathrm{d}_{6} ; \delta$ ), diphosphine: 7.79 $\left(\mathrm{m}\left(\mathrm{A}_{2} \mathrm{X}_{2}\right),\left\{\mathrm{P}-\mathrm{C}_{6} \mathrm{H}_{4}-\mathrm{P}\right\} 12 \mathrm{H},<J(\mathrm{P}-\mathrm{H})>3.6 \mathrm{~Hz}\right), 7.49\left(\mathrm{dm}\left(\mathrm{AXX}^{\prime}\right)\right.$, Pyr 2-CH, 24H, $J(\mathrm{H}-\mathrm{H}) 2.1, J(\mathrm{P}-\mathrm{H}) 4.2 \mathrm{~Hz}), 6.50\left(\mathrm{dm}\left(\mathrm{AXX}^{\prime}\right)\right.$, Pyr 3-CH, 24H, $J(\mathrm{H}-\mathrm{H}) 2.1, J(\mathrm{P}-\mathrm{H}) 2.0 \mathrm{~Hz}) .{ }^{13} \mathrm{C}$ NMR (acetone$\left.\mathrm{d}_{6} ; \delta\right),\left\{\mathrm{Au}\left(\mathrm{C}_{2} \mathrm{C}_{6} \mathrm{H}_{4} \mathrm{OMe}\right)_{2}\right\}: 161.66$ (s, C-para-OMe), 135.58 and 115.35(s, C-ortho and C-meta), 116.58 (s, C-ipso), 115.56 (s, $\mathrm{C} \equiv \mathrm{C}-\mathrm{Au}), 109.85(\mathrm{~s}, \mathrm{C} \equiv \mathrm{C}-\mathrm{Au}) .{ }^{13} \mathrm{C}$ NMR (acetone- $\left.\mathrm{d}_{6} ; \delta\right)$, diphosphine: $137.97\left(\mathrm{~m}\left(\mathrm{AXX} \bar{X}^{\prime}\right), \mathrm{C}\left(-\mathrm{C}_{6} \mathrm{H}_{4}-\right), J(\mathrm{P}-\mathrm{C}) 83.2 \mathrm{~Hz}\right)$, $135.10\left(\mathrm{~m}\left(\mathrm{AXX}^{\prime}\right), \mathrm{C}(\mathrm{H})\left(-\mathrm{C}_{6} \mathrm{H}_{4}-\right), J(\mathrm{P}-\mathrm{C})\right.$ ca. $\left.12 \mathrm{~Hz}\right), 127.78$ (m (AXX'), Pyr 2-C, $J(\mathrm{P}-\mathrm{C})$ ca. $8 \mathrm{~Hz}), 117.37$ (s, Pyr 3-C). Anal. calcd for $\mathrm{C}_{120} \mathrm{H}_{102} \mathrm{Au}_{6} \mathrm{Cu}_{2} \mathrm{~F}_{12} \mathrm{~N}_{12} \mathrm{O}_{6} \mathrm{P}_{8}: \mathrm{C}, 40.12 ; \mathrm{H}, 2.86 ; \mathrm{N}, 4.68$. Found: C, 39.66; H, 2.85; N, 4.50.

Photophysical Measurements. The steady-state absorption and emission spectra were recorded on a Hitachi (U-3310) spectrophotometer and an Edinburgh (FS920) fluorometer, respectively. Both the wavelength-dependent excitation and emission response of the fluorometer have been calibrated. To determine the photoluminescence quantum yield in solution, the samples were degassed by three freeze-pump-thaw cycles. 4-(Dicyanomethylene)-2-methyl6-(paradimethylaminostyryl)-4H-pyran (DCM, $\lambda_{\max }=615 \mathrm{~nm}$, Exciton) in methanol, with a quantum yield of $\sim 0.43$, served as the standard for measuring the quantum yield. ${ }^{10}$ Lifetime studies were performed with an Edinburgh FL 900 photon-counting system using a hydrogen-filled lamp as the excitation source. The data were analyzed using the nonlinear least-squares procedure in combination with an iterative convolution method. The emission decays were analyzed by the sum of the exponential functions, which allows partial removal of the instrument time broadening and consequently renders a temporal resolution of $\sim 300$ ps.

Computational Details. The studied supramolecular $\mathrm{Au}(\mathrm{I})-\mathrm{Cu}(\mathrm{I})$ complexes were fully optimized without any symmetry constraints using the BP86 density functional method. The copper and gold atoms were described with a triple-valence $\zeta$-quality basis set with the polarization functions (def2-TZVP), ${ }^{11}$ employing a 60 -electron relativistic effective core potential for gold. ${ }^{12} \mathrm{~A}$ split-valence basis set with the polarization functions on non-hydrogen atoms was used for all of the other atoms (def2-SV(P)). ${ }^{13}$ The multipole-accelerated resolution-of-the-identity technique was used to speed up the calculations. ${ }^{14}$ All of the geometry optimizations were carried out with TURBOMOLE versions 5.9.1 and 5.10. ${ }^{15}$ The triplet states were studied using spin-unrestricted formalism.

\section{Results and Discussion}

Synthesis and Characterization. Completely analogous to the preparation of the previously described complex $\left[\mathrm{Au}_{2}(\mathrm{C} \equiv \mathrm{CPh})_{2}\left(\mu-1,4-\mathrm{PPh}_{2} \mathrm{C}_{6} \mathrm{H}_{4} \mathrm{PPh}_{2}\right)\right],{ }^{16}$ treatment of the polymers $\left[\mathrm{AuC} \equiv \mathrm{CC}_{6} \mathrm{H}_{4} \mathrm{X}\right]_{n}\left(\mathrm{X}=\mathrm{NO}_{2}, \mathrm{H}, \mathrm{OMe}, \mathrm{NMe}_{2}\right)$ with the diphosphine ligands $\mathrm{PR}_{2} \mathrm{C}_{6} \mathrm{H}_{4} \mathrm{PR}_{2}\left(\mathrm{R}=\mathrm{Ph}, \mathrm{NC}_{4} \mathrm{H}_{4}\right)$ leads to clean formation of the digold complexes $\left[\mathrm{Au}_{2}\left(\mathrm{C} \equiv \mathrm{CC}_{6} \mathrm{H}_{4} \mathrm{X}\right)_{2}(u\right.$ 1,4- $\left.\left.\mathrm{PR}_{2} \mathrm{C}_{6} \mathrm{H}_{4} \mathrm{PR}_{2}\right)\right](2-7)$ in greater than $85 \%$ yield (Scheme 1). These compounds were characterized by ${ }^{1} \mathrm{H}$ and ${ }^{31} \mathrm{P}$ NMR spectroscopy and elemental analysis.

The assembly of the heterometallic $\mathrm{Au}(\mathrm{I})-\mathrm{Cu}(\mathrm{I})$ complexes of the general formula $\left[\left\{\mathrm{Au}_{3} \mathrm{Cu}_{2}\left(\mathrm{C}_{2} \mathrm{C}_{6} \mathrm{H}_{4} \mathrm{X}\right)_{6}\right\} \mathrm{Au}_{3^{-}}\right.$ $\left.\left(\mathrm{PR}_{2} \mathrm{C}_{6} \mathrm{H}_{4} \mathrm{PR}_{2}\right)_{3}\right]\left[\mathrm{PF}_{6}\right]_{2}\left(\mathrm{X}=\mathrm{NO}_{2}, \mathrm{H}, \mathrm{OMe}, \mathrm{NMe}_{2} ; \mathrm{R}=\mathrm{Ph}\right.$, $\mathrm{NC}_{4} \mathrm{H}_{4}$ ) was achieved by reacting the digold complexes $\mathbf{2}-\mathbf{7}$ with stoichiometric amounts of $\mathrm{Cu}(\mathrm{NCMe})_{4} \mathrm{PF}_{6}$ in dichloromethane at room temperature, similarly to the procedure reported earlier (Scheme 2) ${ }^{6}$ Bright-orange to deep-red airstable solids 8-14 were isolated in $\mathbf{7 1 - 9 1 \%}$ yield after recrystallization. Formation of the heterometallic complex with $\mathrm{R}=\mathrm{NC}_{4} \mathrm{H}_{4}$ and $\mathrm{X}=\mathrm{NMe}_{2}(\mathbf{1 5})$ has been observed using ${ }^{1} \mathrm{H}$ and ${ }^{31} \mathrm{P}$ NMR spectroscopy, but it appeared to be unstable, which prevented its isolation in a pure form and subsequent structural and photophysical studies. It is also worth noting that we attempted to prepare analogous heterometallic complexes $\left(\mathrm{X}=\mathrm{NO}_{2}, \mathrm{H}, \mathrm{OMe}, \mathrm{NMe}_{2}\right)$ containing diphosphine

(10) Drake, J. M.; Lesiecki, M. L.; Camaioni, D. M. Chem. Phys. Lett. 1985, 113, 530-534.

(11) Weigend, F.; Ahlrichs, R. Phys. Chem. Chem. Phys. 2005, 7, 32973305.

(12) Andrae, D.; Häußermann, U.; Dolg, M.; Stoll, H.; Preu $\beta$, H. Theor. Chem. Acc. 1990, 77, 123-141.

(13) Schäfer, A.; Horn, H.; Ahlrichs, R. J. Chem. Phys. 1992, 97, 25712577.

(14) (a) Eichkorn, K.; Treutler, O.; Öhm, H.; Häser, M.; Ahlrichs, R. Chem. Phys. Lett. 1995, 240, 283-290. (b) Sierka, M.; Hogekamp, A.; Ahlrichs, R. J. Chem. Phys. 2003, 118, 9136-9148. (c) Eichkorn, K.; Weigend, F.; Treutler, O.; Ahlrichs, R. Theor. Chem. Acc. 1997, 97, 119-124. (d) Weigend, F. Phys. Chem. Chem. Phys. 2006, 8, 10571065.

(15) Ahlrichs, R.; Bär, M.; Häser, M.; Horn, H.; Kölmel, C. Chem. Phys. Lett. 1989, 62, 165-169.

(16) Yam, V. W.-W.; Choi, S. W.-K.; Cheung, K.-K. Organometallics 1996, $15,1734-1739$. 


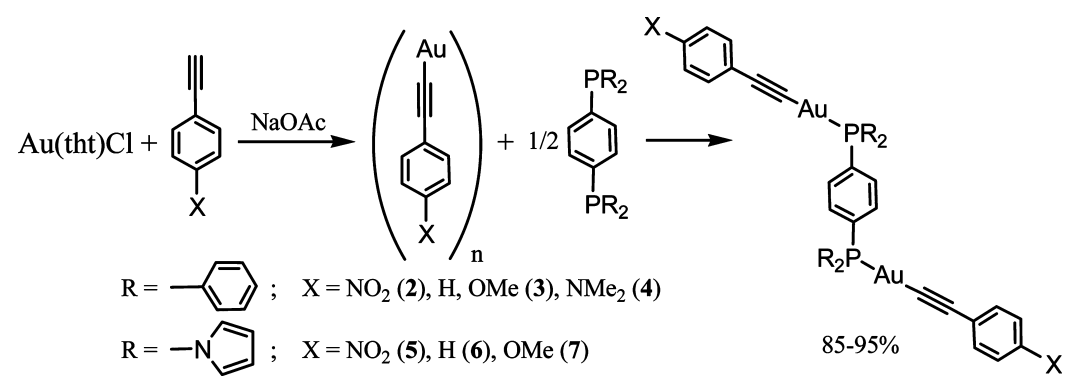

\section{Scheme 2}

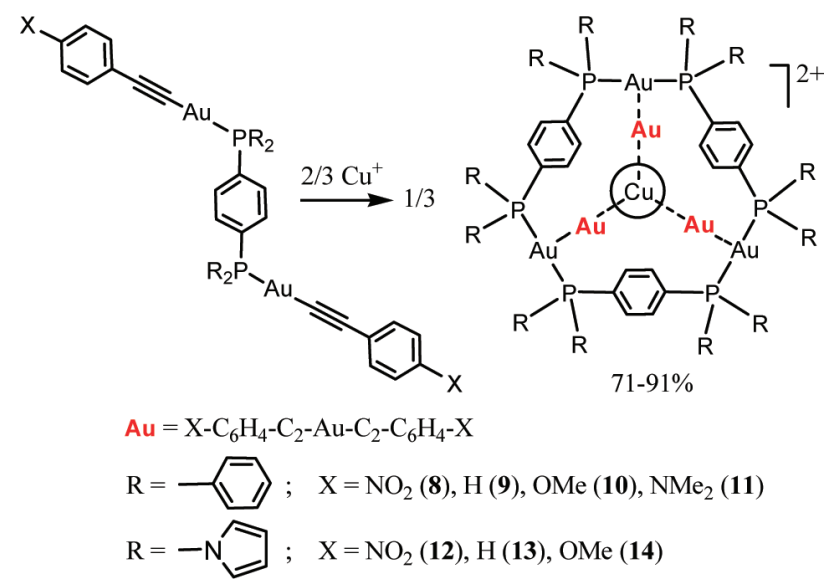

with $\mathrm{R}=i$-Pr using the same synthetic approach. However, no aggregates of the structural type shown in Scheme 2 were formed judging by the NMR analysis of the crude products. The spectroscopic data showed that in these cases only simple coordination of $\mathrm{Cu}^{+}$ions to the $\pi$ orbitals of the alkynyl ligands most probably occurred.

Compounds $8-14$ were completely characterized by ${ }^{1} \mathrm{H}$, ${ }^{13} \mathrm{C}$, and ${ }^{31} \mathrm{P}$ NMR and ESI-MS spectrometry. The crystal structure of complex 9 was elucidated previously in an X-ray diffraction study. ${ }^{6}$

The choice of the alkynyl and phosphine ligands was dictated by their different electron-donor properties. Variation of the substituents in the alkynylbenzenes used in this study from the electron-withdrawing $\mathrm{NO}_{2}$ to the electron-donating $\mathrm{NMe}_{2}$ was aimed to influence electronic characteristics of the central heterometallic fragment. Replacement of the P-bonded phenyls for pyrrolyls in the diphosphine ligand, which are known to be the strong electron-withdrawing groups, results in a dramatic increase of the phosphorus ligand's $\pi$-acidity ${ }^{17}$ This obviously influences the properties of the tricationic $\left[\mathrm{AuPR}_{2}\left(\mathrm{C}_{6} \mathrm{H}_{4}\right)_{2} \mathrm{PR}_{2}\right]_{3}$ "belt", thus making possible variations in the energy of the orbitals, which take part in the formation of the excited states. Therefore, it is sensible to divide the compounds studied into two groups according to the nature of the diphosphine ligand used to wrap the central gold-copper alkynyl fragment. The first group of complexes is based on the $\mathrm{PPh}_{2} \mathrm{C}_{6} \mathrm{H}_{4} \mathrm{PPh}_{2}$ ligand, whereas in the second, the diphosphine contains pyrrolyl substituents at the phosphorus atoms, $\mathrm{P}\left(\mathrm{NC}_{4} \mathrm{H}_{4}\right)_{2} \mathrm{C}_{6} \mathrm{H}_{4} \mathrm{P}\left(\mathrm{NC}_{4} \mathrm{H}_{4}\right)_{2}$.

The ESI mass spectra of compounds 8, 10, and $\mathbf{1 1}$ (Figure S1, S denotes Supporting Information) display signals of the

(17) Moloy, K. G.; Petersen, J. L. J. Am. Chem. Soc. 1995, 117, 76967710 . doubly charged $\quad\left[\left\{\mathrm{Au}_{3} \mathrm{Cu}_{2}\left(\mathrm{C}_{2} \mathrm{C}_{6} \mathrm{H}_{4} \mathrm{X}\right)_{6}\right\} \mathrm{Au}_{3}\left(\mathrm{PPh}_{2} \mathrm{C}_{6} \mathrm{H}_{4^{-}}\right.\right.$ $\left.\left.\mathrm{PPh}_{2}\right)_{3}\right]^{2+}$ molecular ions, the $\mathrm{m} / z$ values and isotopic patterns of which completely match the calculated ones. Comparison of the ${ }^{1} \mathrm{H}$ and ${ }^{13} \mathrm{C}$ NMR data obtained in the previous study for the completely characterized complex $\mathbf{9},{ }^{6}$ with the relative intensities of the signals, their chemical shifts, and coupling constants observed in the spectra of the congeners $8, \mathbf{1 0}$, and 11 containing $\mathrm{NO}_{2}, \mathrm{OMe}$, and $\mathrm{NMe}_{2}$ substituents in the phenyl ring of the alkynyl moieties, clearly showed that these complexes retain the parent structure (Figure 1) in solution.

The NMR parameters of the triangular belts of the substituted congeners are nearly identical (see the Experimental Section) to those of the parent compound, which is also indicative of a weak influence of the alkynyl "rod" substituents on the shielding of the nuclei in the tri(golddiphosphine) belt. The only variations observed in the NMR spectra of these complexes are related to the chemical shifts of the substituted phenyl moieties. The order of the orthoand meta-protons' chemical shifts is clearly related to the donor ability of the corresponding substituents $\left(\mathrm{NMe}_{2}>\right.$ $\mathrm{OMe}>\mathrm{H}>\mathrm{NO}_{2}$ ). It is worth noting that the substituents display a long-range electronic effect, which can be observed in the shielding of the remote alkynyl carbon atoms; for example, high-field shifts of the carbon bound to $\mathrm{Au}$ (109.87 $\operatorname{ppm}\left\{\mathrm{NMe}_{2}\right\}, 110.94 \operatorname{ppm}\{\mathrm{OMe}\}, 112.60 \operatorname{ppm}\{\mathrm{H}\}, 117.39$ $\operatorname{ppm}\left\{\mathrm{NO}_{2}\right\}$ ) very well follow the order of the substituents' donor properties.

According to the elemental analysis and mass spectroscopic data (Figure S1), the other group of compounds, 12-14, based on 1,4-bis(dipyrrolylphosphino)benzene (1) fits

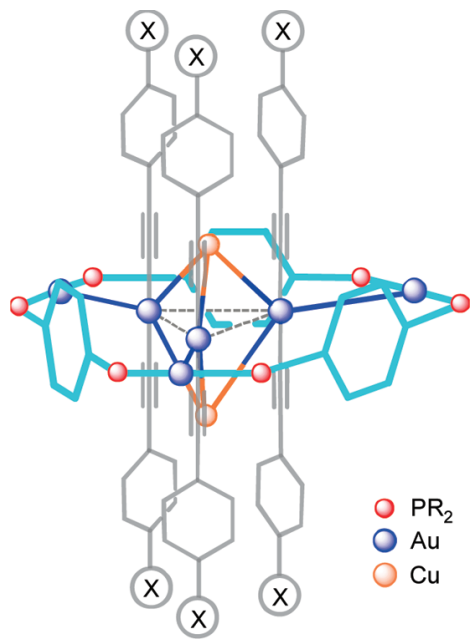

Figure 1. Schematic structure of the dications $\left[\left\{\mathrm{Au}_{3} \mathrm{Cu}_{2}\left(\mathrm{C}_{2} \mathrm{C}_{6} \mathrm{H}_{4} \mathrm{X}\right)_{6}\right\} \mathrm{Au}_{3}-\right.$ $\left.\left(\mathrm{PR}_{2} \mathrm{C}_{6} \mathrm{H}_{4} \mathrm{PR}_{2}\right)_{3}\right]^{2+}$ (8-14), $\left(\mathrm{X}=\mathrm{NO}_{2}, \mathrm{H}, \mathrm{OMe}, \mathrm{NMe}_{2} ; \mathrm{R}=\mathrm{Ph}, \mathrm{NC}_{4} \mathrm{H}_{4}\right)$. 


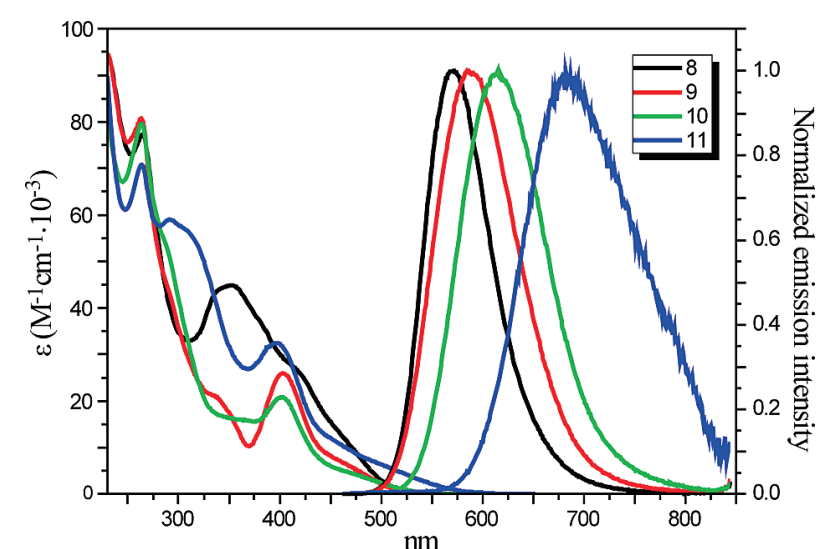

Figure 2. UV/vis absorption and normalized emission spectra of $\mathbf{8}-\mathbf{1 1}$ in deaerated $\mathrm{CH}_{2} \mathrm{Cl}_{2}$ at room temperature, $\lambda_{\text {excit }}=450 \mathrm{~nm}$.

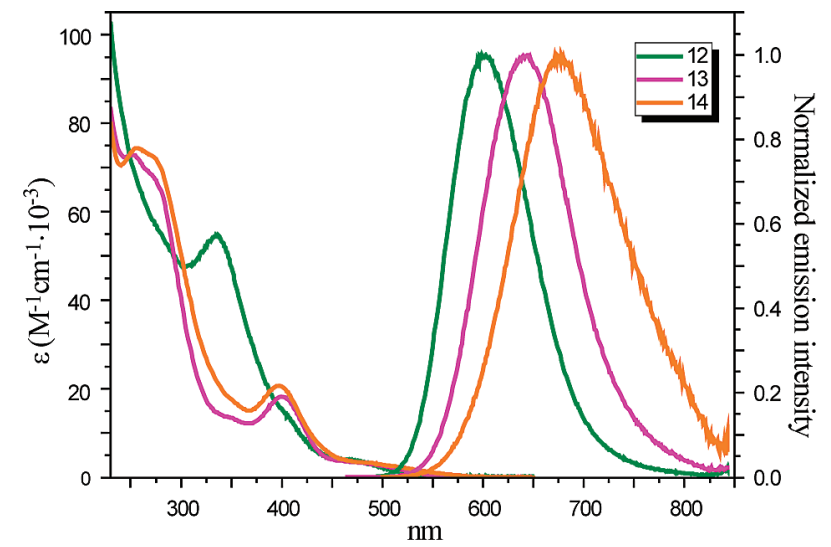

Figure 3. UV/vis absorption and normalized emission spectra of 12-14 in deaerated $\mathrm{CH}_{2} \mathrm{Cl}_{2}$ at room temperature, $\lambda_{\text {excit }}=450 \mathrm{~nm}$.

the stoichiometry of the $\left[\left\{\mathrm{Au}_{3} \mathrm{Cu}_{2}\left(\mathrm{C}_{2} \mathrm{C}_{6} \mathrm{H}_{4} \mathrm{X}\right)_{6}\right\} \mathrm{Au}_{3}\left(\mathrm{P}\left(\mathrm{NC}_{4} \mathrm{H}_{4}\right)_{2^{-}}\right.\right.$ $\left.\left.\mathrm{C}_{6} \mathrm{H}_{4} \mathrm{P}\left(\mathrm{NC}_{4} \mathrm{H}_{4}\right)_{2}\right)_{3}\right]\left[\mathrm{PF}_{6}\right]_{2}$ species. Analysis of the ${ }^{1} \mathrm{H},{ }^{13} \mathrm{C}$, and ${ }^{31} \mathrm{P}$ NMR data for this series of complexes (see the Experimental Section and Figure S2, ${ }^{1} \mathrm{H}$ COSY spectrum of 13) indicates that the components of these polynuclear compounds form structural patterns completely analogous to those of complexes $\mathbf{8}-\mathbf{1 0}$ and contain the central $\left[\mathrm{Au}_{3} \mathrm{Cu}_{2}\left(\mathrm{C}_{2} \mathrm{C}_{6} \mathrm{H}_{4} \mathrm{X}\right)_{6}\right]$ fragments, which are wrapped about by the $\left[\mathrm{AuP}\left(\mathrm{NC}_{4} \mathrm{H}_{4}\right)_{2}\left(\mathrm{C}_{6} \mathrm{H}_{4}\right)_{2} \mathrm{P}\left(\mathrm{NC}_{4} \mathrm{H}_{4}\right)_{2}\right]_{3}$ "belts" anchored to the central parts by $\mathrm{Au}-\mathrm{Au}$ bonds (Figure 1). The presence of only one signal in the ${ }^{31} \mathrm{P}$ spectra of these compounds is indicative of the $D_{3 h}$ symmetry group the $\mathrm{Au}_{3}(\mathrm{PP})_{3}$ belts belong to. The number of signals in the ${ }^{1} \mathrm{H}$ and ${ }^{13} \mathrm{C}$ spectra is also completely compatible with the $D_{3 h}$ structure of the belt and the $\left[\mathrm{Au}_{3} \mathrm{Cu}_{2}\left(\mathrm{C}_{2} \mathrm{C}_{6} \mathrm{H}_{4} \mathrm{X}\right)_{6}\right]$ core, where three equivalent rods form a right triangle with two copper ions in its center above and below the belt plane. Comparison of the corresponding chemical shifts observed in the spectra of these two groups of compounds again indicates a very weak shielding interaction between the "rods" and "belt". The change of phenyl for pyrrolyl substituents in the diphosphine ligands has a nearly negligible and nonsystematic effect on the shielding of the alkynyl carbons disposed most closely (three to four bonds separation) to the phosphorus atoms.

Photophysical Properties. Figures 2 and 3 show the UV/ vis absorption and emission spectra of complexes 8-14 in dichloromethane. The broad, higher-energy absorptions below $270 \mathrm{~nm}$ for all of the titled compounds are ascribed to the intraligand $\pi \rightarrow \pi^{*}(\mathrm{C} \equiv \mathrm{CAr})$ transitions of the alkynyl moieties. This assignment is consistent with the previous reports on the relevant alkynyl analogues, for which the dominant absorption band in the spectral region of 230-300 $\mathrm{nm}$, in general, is ascribed to the characteristic band of the alkynyl-phosphine moieties in combination with their smaller congeners. ${ }^{5,16,18}$ As for the lower-lying transitions, the bands centered at $\sim 300 \mathrm{~nm}$ can be reasonably assigned to an electronic transition from the $\sigma(\mathrm{Au}-\mathrm{P})$ orbital to an empty $\pi^{*}(\mathrm{C} \equiv \mathrm{CPh}$ or phosphine $)$ antibonding orbital located at the bridging phenyl group. ${ }^{16,18}$ The $300-400 \mathrm{~nm}$ absorption bands for these complexes are mainly due to the $\mathrm{Cu}-\pi$-alkynyl fragment metal- and cluster-centered transitions. ${ }^{5,16}$ The lowest energy transition featured with a shoulder and extended to $\sim 500 \mathrm{~nm}$ is likely to possess some metal $(\mathrm{d}$ of $\mathrm{Au}$ and $\mathrm{Cu}) \rightarrow$ alkynes $\left(\pi^{*}\right)$ charge transfer character, but due to the very mixed nature of the HOMO and the LUMO (see computational section), it cannot be described as a pure metal-to-ligand charge-transfer (MLCT) transition.

Remarkably strong luminescence was observed for all of the compounds in dichloromethane. Complexes 8-11 possess the same bridging ligand, $\mathrm{PPh}_{2} \mathrm{C}_{6} \mathrm{H}_{4} \mathrm{PPh}_{2}$, while the difference lies in the variation of the $\mathrm{X}$-substituting group in the para position, as marked in Figure 1. As a result, the emission peak wavelength reveals a bathochromic shift from $\mathbf{8}(576 \mathrm{~nm})$ to $\mathbf{1 1}(686 \mathrm{~nm})$, as shown in Figure 2. Because the electron-donating strength of the $\mathrm{X}$-substituent gradually increases from 8 to 11, the result can be rationalized by the lifting of the HOMO energy level upon increasing the electron density at the alkyne moiety, giving rise to the reduction of the HOMO-LUMO energy gap. Similar results were obtained for the other class of complexes, 12-14, that possess another bridging ligand, $\mathrm{PR}_{2} \mathrm{C}_{6} \mathrm{H}_{4} \mathrm{PR}_{2}\left(\mathrm{R}=\mathrm{NC}_{4} \mathrm{H}_{4}\right)$. Anchoring the substituent in the $\mathrm{X}$ position with increasing electron donating strength from $-\mathrm{NO}_{2}$ to $-\mathrm{OMe}$ causes the emission red shift from $607 \mathrm{~nm}(\mathbf{1 2})$ to $671 \mathrm{~nm}$ (14) (see Figure 3). These results are also consistent with those deduced from the theoretical approach (vide infra). As for the bridging-ligand effect, for example, complexes 8 and $\mathbf{1 2}$, having different bridging ligands but identical $\mathrm{X}$ substituents, exhibit appreciably different emission peak wavelengths (576 $\mathrm{nm}$ for 8 versus $607 \mathrm{~nm}$ for 12). As elaborated in the computational section, the LUMOs of $\mathbf{8}$ and $\mathbf{1 2}$ are partially contributed by the bridging-ligand. Thus, in comparison to the $\mathrm{PPh}_{2} \mathrm{C}_{6} \mathrm{H}_{4} \mathrm{PPh}_{2}$ ligand in $\mathbf{8}$, the stronger electronwithdrawing ligand $\mathrm{PR}_{2} \mathrm{C}_{6} \mathrm{H}_{4} \mathrm{PR}_{2}\left(\mathrm{R}=\mathrm{NC}_{4} \mathrm{H}_{4}\right)$ in $\mathbf{1 2}$ causes greater reduction of the LUMO energy, hence, the reduction of the emission energy gap. A similar argument holds for complexes $\mathbf{9}$ and $\mathbf{1 3}$ as well as for complexes $\mathbf{1 0}$ and $\mathbf{1 4}$.

The quantum yields (Q.Y.), observed lifetimes, and hence the deduced radiative decay rate constants $\left(k_{\mathrm{r}}\right)$ for the titled $\mathrm{Au}-\mathrm{Cu}$ complexes are summarized in Table 1. The mag-

(18) Müller, T. E.; Choi, S. W.-K.; Mingos, D. M. P.; Murphy, D.; Williams, D. J.; Yam, V. W.-W. J. Organomet. Chem. 1994, 484, 209-224. 
Koshevoy et al.

Table 1. Photophysical Properties of the $\mathrm{Au}-\mathrm{Cu}$ Complexes in $\mathrm{CH}_{2} \mathrm{Cl}_{2}$.

\begin{tabular}{|c|c|c|c|c|c|c|}
\hline complex & & $\lambda_{\mathrm{ab}} / \mathrm{nm}\left(10^{-3} \varepsilon / \mathrm{cm}^{-1} \mathrm{M}^{-1}\right)$ & $\lambda_{\mathrm{em}} / \mathrm{nm}^{a}$ & Q.Y. ${ }^{b}$ & $\tau_{\mathrm{obs}} / \mu \mathrm{s}^{a}$ & $k_{\mathrm{r}} / \mathrm{s}^{-1}{ }^{c}$ \\
\hline \multirow[t]{2}{*}{8} & aerated & 265 (77.2), $351(44.8), 408 \mathrm{sh}(28.3)$ & 576 & 0.73 & 3.15 & $1.94 \times 10^{5}$ \\
\hline & degassed & & & 0.93 & 4.80 & \\
\hline 9 & aerated & 263 (80.7), 336sh (21.1), 403 (26.0) & 594 & 0.67 & 3.69 & $1.55 \times 10^{5}$ \\
\hline \multirow[t]{2}{*}{10} & aerated & 264 (79.6), 287sh (54.0), 402 (20.8) & 620 & 0.33 & 2.60 & $1.33 \times 10^{5}$ \\
\hline & degassed & & & 0.77 & 5.78 & \\
\hline 11 & aerated & $264(70.8), 294(58.8), 395(32.5)$ & 686 & 0.04 & 3.10 & $1.04 \times 10^{5}$ \\
\hline 12 & degassed & & & 0.62 & 6.82 & \\
\hline \multirow[t]{2}{*}{13} & aerated & 253 (72.8), 401 (18.3), & 650 & 0.26 & 3.72 & $7.82 \times 10^{4}$ \\
\hline & degassed & & & 0.61 & 7.80 & \\
\hline \multirow[t]{2}{*}{14} & aerated & 260 (74.1), 398 (20.7) & 671 & 0.12 & 1.61 & $7.22 \times 10^{4}$ \\
\hline & degassed & & & 0.19 & 2.69 & \\
\hline
\end{tabular}

${ }^{a} \lambda_{\text {excit }}=450 \mathrm{~nm} .{ }^{b}$ Measured in dichloromethane solutions; DCM in methanol was used as a standard for the quantum yield measurements. ${ }^{c} k_{\mathrm{r}}$ is deduced from data obtained in the degassed solution. ${ }^{d}$ This value is slightly higher than that given in ref 6 , but both are within the experimental error $(8-10 \%)$.

Table 2. Selected Structural Parameters and Frontier Orbital Characteristics of the Substituted $\mathrm{Au}(\mathrm{I})-\mathrm{Cu}(\mathrm{I}) \mathrm{Complexes}$ 8-15

\begin{tabular}{|c|c|c|c|c|c|c|c|c|}
\hline & 8 & 9 & 10 & 11 & 12 & 13 & 14 & 15 \\
\hline $\mathbf{X}$ & $\mathrm{NO}_{2}$ & $\mathrm{H}$ & $\mathrm{OMe}$ & $\mathrm{NMe}_{2}$ & $\mathrm{NO}_{2}$ & $\mathrm{H}$ & $\mathrm{OMe}$ & $\mathrm{NMe}_{2}$ \\
\hline $\mathbf{R}$ & $\mathrm{Ph}$ & $\mathrm{Ph}$ & $\mathrm{Ph}$ & $\mathrm{Ph}$ & $\mathrm{NC}_{4} \mathrm{H}_{4}$ & $\mathrm{NC}_{4} \mathrm{H}_{4}$ & $\mathrm{NC}_{4} \mathrm{H}_{4}$ & $\mathrm{NC}_{4} \mathrm{H}_{4}$ \\
\hline \multicolumn{9}{|c|}{ Structural Parameters Shown in Figure $4(\AA)^{a}$} \\
\hline $\mathrm{R}(\mathrm{Au}-\mathrm{Au}(\mathrm{c}-\mathrm{c}))$ & 3.40 & $3.39(3.33-3.36)$ & 3.39 & 3.39 & 3.39 & 3.37 & 3.37 & 3.35 \\
\hline $\mathrm{R}(\mathrm{Au}-\mathrm{Cu})$ & 2.81 & $2.82(2.75-2.91)$ & 2.81 & 2.80 & 2.83 & 2.83 & 2.81 & 2.80 \\
\hline $\mathrm{R}(\mathrm{Au}-\mathrm{C})$ & 2.03 & $2.03(1.97-2.01)$ & 2.03 & 2.03 & 2.02 & 2.02 & 2.03 & 2.03 \\
\hline \multicolumn{9}{|c|}{ Frontier Orbital Characteristics $(\mathrm{eV})$} \\
\hline HOMO-LUMO gap & 1.82 & 1.80 & 1.63 & 1.38 & 1.66 & 1.45 & 1.17 & 0.87 \\
\hline HOMO & -8.70 & -7.80 & -7.35 & -6.76 & -9.17 & -8.32 & -7.77 & -7.12 \\
\hline LUMO & -6.88 & -6.00 & -5.72 & -5.38 & -7.51 & -6.87 & -6.60 & -6.24 \\
\hline
\end{tabular}

${ }^{a}$ Average bond lengths. ${ }^{b}$ For complex 9 , the experimental structural parameters are listed in parentheses.

nitudes of the observed lifetimes of microseconds and the radiative decay rate constant of $\sim 10^{5} \mathrm{~s}^{-1}$ lead us to conclude that the emission originates from the triple state manifold, that is, the phosphorescence. A striking feature of these complexes lies in that the phosphorescence intensity is subject to minor $\mathrm{O}_{2}$ quenching in aerated solutions. For example, the Q.Y. of 8 (in $\mathrm{CH}_{2} \mathrm{Cl}_{2}$ ) was measured to be 0.93 $(4.80 \mu \mathrm{s})$ in a degassed solution, while to our surprise, Q.Y. only decreased to 0.73 with an observed lifetime as long as $3.15 \mu \mathrm{s}$. On the basis of the collisional type of energy transfer, the rate of $\mathrm{O}_{2}$ quenching to the triplet state is approximately $1 / 9$ of the (solvent) diffusion-controlled rate. Accordingly, in the aerated solution, the observed lifetime of phosphorescence is commonly an order of magnitude lower, dropping the value to a few hundred nanoseconds. ${ }^{19}$ The high quantum yield and hence rather small $\mathrm{O}_{2}$ quenching rate manifest the uniqueness of the framework built by these titled supramolecules, in which the emission chromophores, that is, metal and alkynyl groups, are largely protected by the bulky ancillary and bridging ligands (see Figure 1). This unique scaffold greatly prevents $\mathrm{O}_{2}$ collision and hence the reduction of the $\mathrm{O}_{2}$ quenching rate. The nearly $\mathrm{O}_{2-}$ independent phosphorescence is remarkable and should render ample applications in, for example, phosphorescence dyes in time-resolved imaging. ${ }^{20}$

Computational Results. Density functional calculations were performed to provide additional insight into the

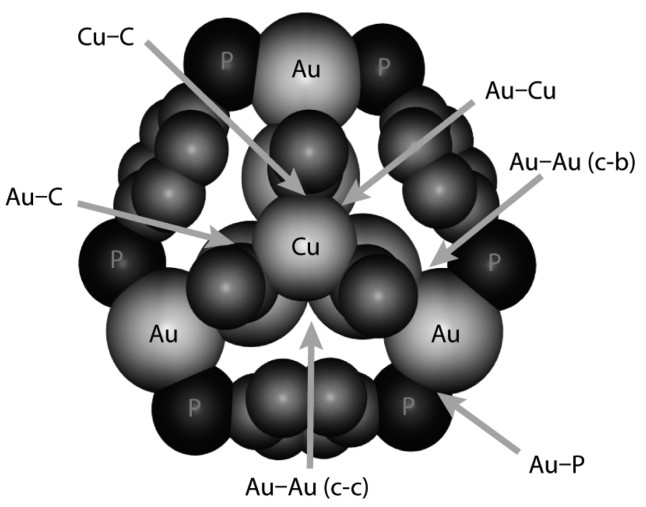

Figure 4. Optimized geometry of complex 9, showing the structural parameters listed in Table 2 for all of the studied complexes. The complex is shown from a top view, omitting phenyl rings and hydrogen atoms for clarity.

structural and electronic properties of the supramolecular $\mathrm{Au}(\mathrm{I})-\mathrm{Cu}(\mathrm{I})$ complexes (for computational details, see the Experimental Section). The computational results are summarized in Table 2 and Figure 4. In the case of complex 9, the availability of the X-ray crystal structure enabled the comparison between the theoretical and experimental geometries. The DFT-optimized and experimental structural parameters were found to be in good agreement. All of the studied complexes $\mathbf{8}-\mathbf{1 5}$ retain the "rods-in-belt" structural motif during the geometry optimization, and the changes in structural parameters are fairly small. Some structural trends can be observed, however. As $\mathrm{X}$ is changed from electronwithdrawing to electron-donating, the $\mathrm{Au}-\mathrm{Au}$ bond contacts 


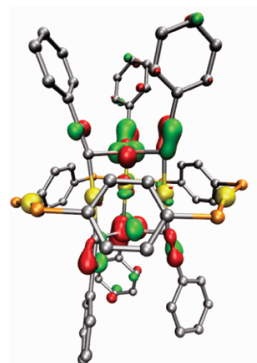

$\mathrm{S}_{0} \mathrm{HOMO}$

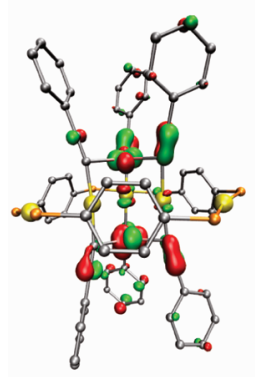

$\mathrm{S}_{0}$ HOMO

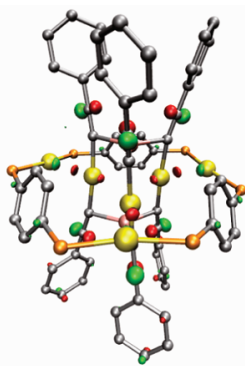

SO LUMO

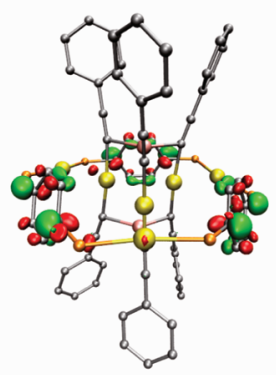

$S_{0}$ LUMO

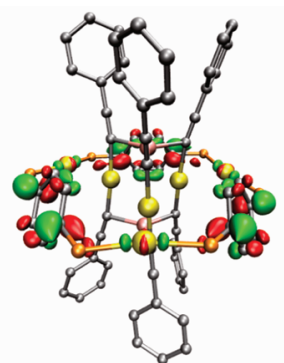

$S_{0}$ LUMO + 1

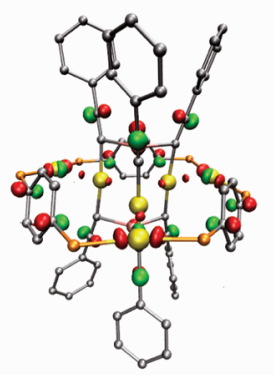

$S_{0}$ LUMO + 1

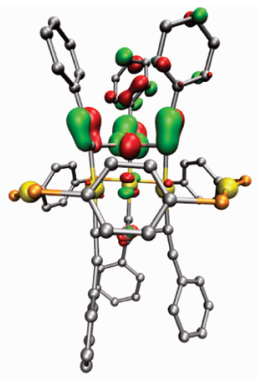

$\mathrm{T}_{1}$ LSOMO

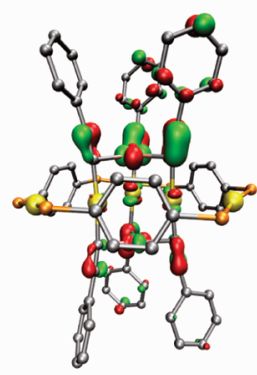

$\mathrm{T}_{1}$ LSOMO
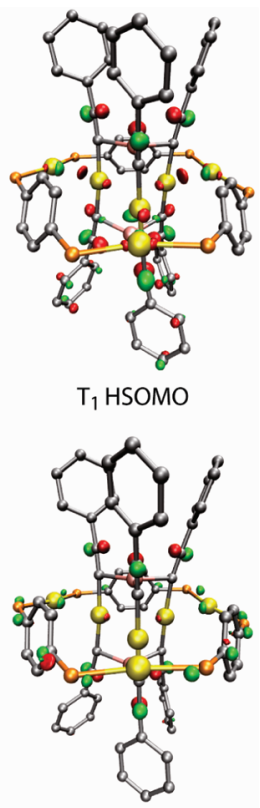

$\mathrm{T}_{1}$ HSOMO

Figure 5. Selected frontier molecular orbital isodensity plots for the $\mathrm{Au}(\mathrm{I})-\mathrm{Cu}(\mathrm{I})$ complexes $\mathbf{9}$ (top) and $\mathbf{1 3}$ (bottom). Phenyl (9) and pyrrolyl rings (13) of the "belt" fragment, and all hydrogen atoms omitted for clarity. Isodensity value of 0.04 au was used for all plots. SOMO = singly occupied molecular orbital.

between the central and "belt" fragments become shorter. $\mathrm{The} \mathrm{Au}-\mathrm{Au}$ and $\mathrm{Au}-\mathrm{Cu}$ distances in the central fragment show a similar decreasing trend. Because the electrondonating substituents in the $\mathrm{X}$ position contribute additional electron density to the central fragment, the Coulombic attraction between the anionic central fragment and the cationic "belt" fragment increases, shortening the supramolecular bond contacts. The structural effect of changing $\mathrm{R}$ from $\mathrm{Ph}$ to $\mathrm{NC}_{4} \mathrm{H}_{4}$ is less significant, although a slight shortening of the $\mathrm{Au}-\mathrm{Au}$ distances can be observed.

The frontier molecular orbitals of the $S_{0}$ and $T_{1}$ electronic states of complexes $\mathbf{9}$ and $\mathbf{1 3}(\mathrm{X}=\mathrm{H})$ are illustrated in Figure 5. In both complexes, HOMOs are located within the central fragment, the most significant contributions coming from the $\mathrm{d}(\mathrm{Cu}), \pi(\mathrm{C} \equiv \mathrm{CPh})$, and $\mathrm{d}(\mathrm{Au})$ orbitals. In complex $9(\mathrm{R}=$ $\mathrm{Ph})$, the main contributions to the LUMO come from central fragment $\mathrm{sp}(\mathrm{Au}), \pi^{*}(\mathrm{C} \equiv \mathrm{CPh})$, and $\mathrm{sp}(\mathrm{Cu})$ orbitals. The LUMO+1 of 9 is delocalized over the "belt" fragment, with the largest contributions coming from the bridging Ph groups. Changing X from electron-withdrawing into electron-donating affects the frontier orbitals mainly by increasing the contribution of the "belt" fragment on the LUMO. Changing $\mathrm{R}$ from $\mathrm{Ph}$ to $\mathrm{NC}_{4} \mathrm{H}_{4}$ further increases the contribution of the "belt" fragment to the LUMOs, practically reversing their ordering in comparison to 9. For all studied complexes, geometry optimization of the lowest-lying triplet state results

(19) (a) Tung, Y.-L.; Wu, P.-C.; Liu, C.-S.; Chi, Y.; Yu, J.-K.; Hu, Y.-H.; Chou, P.-T.; Peng, S.-M.; Lee, G.-H.; Tao, Y.; Carty, A. J.; Shu, C.F.; Wu, F.-I. Organometallics 2004, 23, 3745-3748. (b) Hwang, F.M.; Chen, H.-Y.; Chen, P.-S.; Liu, C.-S.; Chi, Y.; Shu, C.-F.; Wu, F.-I.; Chou, P.-T.; Peng, S.-M.; Lee, G.-H. Inorg. Chem. 2005, 44, 1344-1353. (c) Tung, Y.-L.; Lee, S.-W.; Chi, Y.; Chen, L.-S.; Shu, C.-F.; Wu, F.-I.; Carty, A. J.; Chou, P.-T.; Peng, S.-M.; Lee, G.-H. Adv. Mater. 2005, 17, 1059-1064.

(20) Hanaoka, K.; Kikuchi, K.; Kobayashi, S.; Nagano, T. J. Am. Chem. Soc. 2007, 129, 13502-13509. in minor structural changes mainly within the $\mathrm{Au}-\mathrm{Au}$, $\mathrm{Au}-\mathrm{Cu}$, and $\mathrm{Cu}-\mathrm{C}$ distances of the central fragment, but the "rods-in-belt" structural motif in general remains unperturbed. For complexes with $\mathrm{R}=\mathrm{Ph}$, the $\mathrm{Au}-\mathrm{Au}$ bond contacts between the central and "belt" fragments shorten slightly, and the highest singly occupied molecular orbital (HSOMO) of the $T_{1}$ state is closely related to the central fragment-based $\mathrm{S}_{0} \mathrm{LUMO}$. For complexes with $\mathrm{R}=\mathrm{NC}_{4} \mathrm{H}_{4}$, the $\mathrm{Au}-\mathrm{Au}$ bond contacts between the central and "belt" fragments are slightly elongated, and the $\mathrm{T}_{1}$ HSOMO is related to the $\mathrm{S}_{0} \mathrm{LUMO}+1$. The lowest singly occupied molecular orbital of the $T_{1}$ state is related to the $\mathrm{S}_{0} \mathrm{HOMO}$ for both $\mathrm{R}=\mathrm{Ph}$ and $\mathrm{R}=\mathrm{NC}_{4} \mathrm{H}_{4}$.

Considering the strong contribution of the heterometallic core to the frontier orbitals of the $\mathrm{S}_{0}$ and $\mathrm{T}_{1}$ states, the longwavelength phosphorescence observed for the complexes is likely to involve a triplet state with a higher-energy SOMO of predominantly metal sp character and a lower-energy SOMO of mixed metal $\mathrm{d}$ and alkynyl $\pi$ character. The initial excited states, such as MLCT $\left[\mathrm{d}(\mathrm{Cu}, \mathrm{Au}) \rightarrow \pi^{*}(\mathrm{C} \equiv \mathrm{Ph})\right]$, end up in the emitting triplet state due to efficient intersystem crossing. ${ }^{6}$ For complexes with $\mathrm{R}=\mathrm{NC}_{4} \mathrm{H}_{4}$, MLCT excitations to "belt" ligands should be considered, as well. When $\mathrm{R}=\mathrm{NC}_{4} \mathrm{H}_{4}$, or $\mathrm{X}$ is an electron-donating substituent, a larger contribution of the less protected "belt" fragment to the excited states might be one reason for the smaller quantum yields observed for such complexes.

The relative energies of the frontier orbitals are significantly altered by the modification of $\mathrm{X}$ and $\mathrm{R}$ (Table 2). As $\mathrm{X}$ changes from electron-withdrawing to electron-donating, the energy of the HOMO increases more than the energy of the LUMO, decreasing the magnitude of the HOMO-LUMO gap. The influence of changing the $\mathrm{R}$ is opposite of the effect of changing $\mathrm{X}$, the more electron-withdrawing $\mathrm{NC}_{4} \mathrm{H}_{4}$ 
decreasing the HOMO-LUMO gap in comparison to $\mathrm{Ph}$. In this case, the decrease of the HOMO-LUMO gap is due to the energy of the LUMO decreasing more than the energy of the central fragment-based HOMO. The magnitude of the calculated HOMO-LUMO gaps decreases in the order $\mathbf{8}>$ $9>12>10>13>11>14$. The decreasing trend of the HOMO-LUMO gaps correlates well with the experimentally observed red-shift of the emission, except for complex 11, which was found to emit at slightly lower energy than complex 14. The HOMO-LUMO gaps calculated for the complexes also provide a qualitative estimate of their kinetic stability. For instance, experimental isolation of the complex where $\mathrm{R}=\mathrm{NC}_{4} \mathrm{H}_{4}$ and $\mathrm{X}=\mathrm{NMe}_{2}$ (15), which has a considerably smaller HOMO-LUMO gap in comparison to other studied complexes, proved to be unsuccessful due to its instability toward decomposition.

Overall, the computational results suggest that, while modifying $\mathrm{X}$ or $\mathrm{R}$ has little effect on the structural properties of the supramolecular $\mathrm{Au}(\mathrm{I})-\mathrm{Cu}(\mathrm{I})$ complexes, their electronic properties are significantly altered by the modification of the substituents. Investigation of the electronic characteristics suggests that a metal-centered triplet emission within the central $\mathrm{Au}(\mathrm{I})-\mathrm{Cu}(\mathrm{I})$-alkynyl fragment plays a key role in the observed long-wavelength phosphorescence.

\section{Conclusion}

We have presented a systematic synthetic, photophysical, and theoretical study of a family of intensely luminescent heterometallic clusters of the general formula $\left[\left\{\mathrm{Au}_{3} \mathrm{Cu}_{2^{-}}\right.\right.$ $\left.\left.\left(\mathrm{C}_{2} \mathrm{C}_{6} \mathrm{H}_{4} \mathrm{X}\right)_{6}\right\} \mathrm{Au}_{3}\left(\mathrm{PR}_{2} \mathrm{C}_{6} \mathrm{H}_{4} \mathrm{PR}_{2}\right)_{3}\right]\left[\mathrm{PF}_{6}\right]_{2}\left(\mathrm{X}=\mathrm{NO}_{2}, \mathrm{H}, \mathrm{OMe}\right.$, $\left.\mathrm{NMe}_{2} ; \mathrm{R}=\mathrm{C}_{6} \mathrm{H}_{5}, \mathrm{NC}_{4} \mathrm{H}_{4}\right)$. These complexes adopt the same structural pattern and consist of heterometallic alkynyl clusters $\left[\mathrm{Au}_{3} \mathrm{Cu}_{2}\left(\mathrm{C}_{2} \mathrm{C}_{6} \mathrm{H}_{4} \mathrm{X}\right)_{6}\right]^{-}$"wrapped" by cationic $\left[\mathrm{Au}_{3}\left(\mathrm{PR}_{2} \mathrm{C}_{6} \mathrm{H}_{4} \mathrm{PR}_{2}\right)_{3}\right]^{3+}$ "belts". All of the compounds under study exhibit strong room-temperature phosphorescence in solution with a maximum quantum yield of 0.96 . The emission maxima wavelength reveals a bathochromic shift upon (a) the increase of the electron-donating strength of the $\mathrm{X}$ substituents of the alkynyl ligands and (b) the increase of the electron-withdrawing strength of the $\mathrm{R}$ groups of the diphosphines. The remarkable feature of these complexes consists of a very low degree of phosphorescence quenching by $\mathrm{O}_{2}$ that is evidently determined by the uniqueness of their framework, in which the emission chromophores, that is, metal and alkynyl groups, are protected by the bulky ancillary and bridging ligands. The theoretical calculations of the electronic structures showed that modifying X or R substituents has little effect on the structural features but significantly influences the electronic properties. The frontier orbital characteristics of the complexes suggest that the observed long-wavelength phosphorescence is associated with metalcentered triplet emission within the heterometallic alkynyl cluster.

Acknowledgment. Financial support from Academy of Finland (I.O.K.) and Russian Foundation for Basic Research (grant 07-03-00908-a) is acknowledged.

Supporting Information Available: ESI-mass spectra of 8, 10-14; ${ }^{1} \mathrm{H}-{ }^{1} \mathrm{H}$ COSY spectrum of 13; and optimized Cartesian coordinates of the studied systems in atomic units. This material is available free of charge via the Internet at http://pubs.acs.org.

IC801987T 